\title{
Immunoproteomic identification of MbovP579, a promising diagnostic biomarker for serological detection of Mycoplasma bovis infection
}

\author{
Farhan Anwar Khan ${ }^{1,2,3, *}$, Muhammad Faisal',2,*, Jin Chao ${ }^{1,2}$, Kai Liu' ${ }^{1,2}$, Xi Chen², \\ Gang Zhao ${ }^{1,2}$, Harish Menghwar ${ }^{1,2}$, Hui Zhang ${ }^{1,2}$, Xifang Zhu ${ }^{1,2}$, Muhammad Asif \\ Rasheed ${ }^{1,2}$, Chenfei $\mathrm{He}^{1,2}$, Changmin $\mathrm{Hu}^{2}$, Yingyu Chen ${ }^{1,4}$, Eric Baranowski ${ }^{6,7}$, \\ Huanchun Chen ${ }^{1,2,4}$ and Aizhen Guo ${ }^{1,2,4,5}$ \\ ${ }^{1}$ The State Key Laboratory of Agricultural Microbiology, Huazhong Agricultural University, Wuhan, China \\ ${ }^{2}$ College of Veterinary Medicine, Huazhong Agricultural University, Wuhan, China \\ ${ }^{3}$ Department of Animal Health, The University of Agriculture, Peshawar, Pakistan \\ ${ }^{4}$ Key Laboratory of Development of Veterinary Diagnostic Products, Ministry of Agriculture, International Joint Research and \\ Training Centre for Veterinary Epidemiology, Huazhong Agricultural University, Wuhan, China \\ ${ }^{5}$ International Joint Research Centre for Veterinary Epidemiology, Huazhong Agricultural University, Wuhan, China \\ ${ }^{6}$ INRA, UMR 1225, IHAP, Toulouse, France \\ 7 INP-ENVT, UMR 1225, IHAP, Université de Toulouse, Toulouse, France \\ * These authors have contributed equally to this work \\ Correspondence to: Aizhen Guo, email: aizhen@mail.hzau.edu.cn \\ Keywords: diagnostic biomarker, ELISA, immunoproteomics, immunoinformatics, Mycoplasma bovis, Immunology and Microbiol- \\ ogy Section, Immune response, Immunity \\ Received: April 27, $2016 \quad$ Accepted: May 23, $2016 \quad$ Published: June 02, 2016
}

\section{ABSTRACT}

A lack of knowledge regarding the antigenic properties of Mycoplasma bovis proteins prevents the effective control of bovine infections using immunological approaches. In this study, we detected and characterized a specific and sensitive M. bovis diagnostic biomarker. After $M$. bovis total proteins and membrane fractions were separated with two dimensional gel electrophoresis, proteins reacting with antisera were detected using MALDI-TOF MS. Thirty-nine proteins were identified, 32 of which were previously unreported. Among them, immunoinformatics predicted eight antigens, encoded by Mbov_0106, 0116, 0126, 0212, 0275, 0579, 0739, and 0789 , to have high immunological value. These genes were expressed in $E$. coli after mutagenesis of UGA to UGG using overlap extension PCR. A lipoprotein, MbovP579, encoded by a functionally unknown gene, was a sensitive and specific antigen for detection of antibodies in sera from both $M$. bovis-infected and vaccinated cattle. The specificity of MbovP579 was confirmed by its lack of cross-reactivity with other mycoplasmas, including Mycoplasma agalactiae. An iELISA based on rMbovP579 detected seroconversion 7 days post-infection (dpi). The ELISA had sensitivity of 90.2\% (95\% CI: $83.7 \%, 94.3 \%)$ and a specificity of $97.8 \%$ (95\% CI: $88.7 \%, 99.6 \%)$ with clinical samples. Additional comparative studies showed that both diagnostic and analytic sensitivities of the ELISA were higher than those of a commercially available kit $(p<0.01)$. We have thus detected and characterized the novel antigen, MbovP579, and established an rMbovP579-based ELISA as a highly sensitive and specific method for the early diagnosis of $M$. bovis infection. 


\section{INTRODUCTION}

Mycoplasma bovis (M. bovis) belongs to Mollicutes, a class of simple self-replicating bacteria characterized by very small genomes ( 580 to $2200 \mathrm{~kb}$ ) and the lack of a cell wall. It is a major pathogen that affects cattle worldwide, causing pneumonia, mastitis, arthritis, keratoconjunctivitis, and otitis $[1,2]$. In Europe, it is responsible for about a quarter of all calf pneumonia cases, causing annual losses estimated at 140 million Euros. The economic impact of $M$. bovis in the USA is similar due to mastitis and respiratory infections [1,3]. Since 2008, $M$. bovis has been reported as a serious threat to the growing beef and dairy industry in China $[4,5]$.

Currently, the primary methods for controlling $M$. bovis are management practices and antimicrobial treatments [2,3]. However, M. bovis is naturally resistant to antimicrobial agents targeting the cell wall, and several studies have reported low susceptibility to many commercially available antimicrobials and the emergence of resistant strains worldwide $[6,7,8,9,10]$. Therefore, our laboratory recently developed an effective live, attenuated vaccine for the control of M. bovis [3]. In vivo and in vitro studies have revealed that both virulent and avirulent strains of $M$. bovis are characterized by genoplasticity and phenotypic diversity $[4,11]$. It is therefore important to identify and characterize antigenic proteins associated with $M$. bovis infection in both virulent strains and attenuated vaccine strains to devise an effective control strategy.

Considerable efforts have been made to elucidate antigenic structures in M. bovis, and some relevant proteins have been identified. They include exposed surface proteins, such as variable surface proteins (Vsp), the lipoproteins P26, P48-like, and mycoplasma immunogenic lipase A (MilA), and the immunodominant membrane protein pMB67 [12, 13, 14, 15, 16, 17], as well as cytoplasmic proteins, such as heat-shock protein 60 (Hsp60), glyceraldehyde-3-phosphate dehydrogenase (GAPDH), and the pyruvate dehydrogenase beta (PDHB) subunit, among others $[18,19,20]$. The highly-conserved M. bovis GAPDH was suggested as a potential antigen for diagnosis or vaccines; however, a subunit vaccine based on GAPDH did not protect against M. bovis [21]. The cause of this poor protective efficacy is unknown, but the host Th2 response, perhaps accompanied by high levels of the weak opsonin IgG1, has been suggested [22]. In general, early diagnosis would assist in the control of $M$. bovis infection in feedlots and dairy herds. Serodiagnostic assays, which might detect the IgG specific to $M$. bovis even in chronically infected cattle or animals exposed to antimicrobial agents, may be particularly helpful in this regard [20]. Although many serodiagnostic assays have been developed $[5,17,20]$, improved serodiagnostic assays based on more sensitive and specific antigens are still required for the early detection of the M. bovis- specific IgG in exposed cattle populations.

Because mycoplasmas lack cell walls, the cell membrane and membrane-associated proteins (MAPs) are important for mycoplasma colonization and survival within hosts. Many lipoproteins are reportedly linked to the cytoplasmic membrane, and the majority are thought to be exposed on the cell surface. However, only a few lipoproteins have been associated with virulence or antigenicity [23]. Therefore, highly conserved, sensitive, and specific antigenic membrane molecules or MAPs may be the most promising targets for establishing novel serodiagnostics.

Proteomics approaches, such as dimensional gel electrophoresis (2-DE) and MALDI-TOF mass spectrometry (MS), have increasingly been used in immunoproteomics studies of several mycoplasma species [20, 24, 25, 26, 27]. However, these data alone are often not enough to identify potential antigens containing multiple T- and B-cell epitopes [28]. Recently, immunoinformatics, a combination of immunology and informatics, has helped elucidate the networks involved in defense system activation [29]. Such methods have been used to successfully identify antigenic epitopes in several pathogens, such as Mycobacterium tuberculosis and Neisseria meningitidis [30,31], and to select potential candidates for serodiagnostics and vaccine development $[32,33]$. Analyses based on murine immunological databases may not apply to other species, including bovines. However, a combination of immunoproteomics, immunoinformatics, conventional gene expression, and subsequent immunological confirmation provides an effective method for comprehensively characterizing antigenic proteins [28].

This study was conducted to assemble a global antigenic profile for $M$. bovis using immunoproteomics and immunoinformatics and to identify promising candidate proteins in $M$. bovis using gene expression analyses and other serological methods. Among the eight identified M. bovis antigens expressed in E. coli, MbovP579 was confirmed as a conserved, sensitive, and specific antigen in both field isolates and the attenuated vaccine strain $M$. bovis-150. An indirect ELISA (iELISA) based on recombinant MbovP579 (rMbovP579) was able to effectively detect the M. bovis-specific IgG in animals with acute or chronic infections.

\section{RESULTS}

\section{Analysis of whole cell proteins (WCPs) from $M$. bovis $\mathrm{HB} 0801$}

Immunoproteomics revealed antigenicity of both membrane-associated and cytoplasmic proteins in the WCPs of M. bovis HB0801 cells. Analysis of M. bovis 
Table 1: Antigenic proteins identified in M. bovis HB0801 whole cell proteins

\begin{tabular}{|c|c|c|c|c|c|c|c|c|c|c|}
\hline $\begin{array}{l}\text { Spot } \\
\text { no. }\end{array}$ & Gene & Protein & NCBI ID & $\begin{array}{l}\text { Mw } \\
\text { (kDa) }\end{array}$ & pI & $\begin{array}{l}\text { Protein } \\
\text { Scores }\end{array}$ & $\begin{array}{l}\text { Protein } \\
\text { Score } \\
\text { C.I.\%* }\end{array}$ & $\begin{array}{l}\text { PSORTb } \\
\text { Localization }\end{array}$ & $\begin{array}{l}\text { PSORTb } \\
\text { Probability }\end{array}$ & COG** \\
\hline 18 & Mbov_0016 & $\begin{array}{l}\text { P48-like } \\
\text { lipoprotein }\end{array}$ & gi|392051019| & 51.2 & 8.8 & 816 & 100 & \begin{tabular}{|l} 
Outer \\
Membrane
\end{tabular} & 9.52 & $\mathrm{R}$ \\
\hline 20 & Mbov_0070 & \begin{tabular}{|l} 
Putative \\
transmembrane \\
protein
\end{tabular} & gi|392051070| & 37.6 & 7.0 & 271 & 100 & $\begin{array}{l}\text { Cytoplasmic } \\
\text { Membrane }\end{array}$ & 10.00 & - \\
\hline 20 & Mbov_0071 & $\begin{array}{l}\text { Aspartate- } \\
\text { ammonia ligase }\end{array}$ & gi|392051071| & 37.8 & 7.1 & 141 & 100 & Cytoplasmic & 10.00 & E \\
\hline 7 & Mbov_0103 & $\begin{array}{l}\text { Pyruvate } \\
\text { dehydrogenase } \\
\text { beta subunit }\end{array}$ & gi|392051103| & 36.1 & 5.4 & 96 & 99.8 & Cytoplasmic & 9.97 & $\mathrm{C}$ \\
\hline $7-8$ & Mbov_0106 & $\begin{array}{l}\text { Dihydrolipoamide } \\
\text { dehydrogenase } \\
\text { (DLD) }\end{array}$ & gi|392051106| & 58.9 & 6.3 & 274 & 100 & Cytoplasmic & 9.97 & $\mathrm{C}$ \\
\hline 8 & Mbov_0212 & $\begin{array}{l}\text { Transketolase } \\
\text { (Tkt) } \\
\end{array}$ & gi|392051206| & 72.5 & 6.1 & 87 & 98.7 & Cytoplasmic & 9.97 & G \\
\hline 14 & Mbov_0304 & $\begin{array}{l}\text { Glycyl-tRNA } \\
\text { synthetase }\end{array}$ & gi|392051297| & 53.0 & 6.0 & 82 & 96.4 & Cytoplasmic & 10.00 & $\mathrm{~J}$ \\
\hline 19 & Mbov_0306 & $\begin{array}{l}\text { Phosphonate } \\
\text { ABC transporter } \\
\text { substrate-binding } \\
\text { protein }\end{array}$ & gi|392051299| & 49.7 & 6.9 & 171 & 100 & $\begin{array}{l}\text { Cytoplasmic } \\
\text { Membrane }\end{array}$ & 9.97 & $\mathrm{P}$ \\
\hline 7 & Mbov_0481 & $\begin{array}{l}\text { Elongation factor } \\
\mathrm{Tu}\end{array}$ & gi|392051462| & 43.6 & 5.8 & 64 & 52.5 & Cytoplasmic & 9.64 & $\mathrm{~J}$ \\
\hline $\begin{array}{l}11- \\
12- \\
13-14 \\
\end{array}$ & Mbov_0508 & $\begin{array}{l}\mathrm{F}_{0} \mathrm{~F}_{1} \text { ATP } \\
\text { subunthase } \\
(\text { AtpD) }\end{array}$ & gi|392051487| & 50.7 & 5.5 & 273 & 100 & $\begin{array}{l}\text { Cytoplasmic } \\
\text { Membrane }\end{array}$ & 9.51 & $\mathrm{C}$ \\
\hline $\begin{array}{l}3-4-5- \\
6-7-8- \\
9-10\end{array}$ & Mbov_0579 & $\begin{array}{l}\text { Lipoprotein } \\
\text { (MbovP579) }\end{array}$ & gi|392051558| & 81.6 & 9.0 & 631 & 100 & Unknown & - & - \\
\hline 2 & Mbov_0628 & \begin{tabular}{|l|}
$50 \mathrm{~S}$ ribosomal \\
protein L4
\end{tabular} & | gi|392051604| & 32.4 & 10.2 & 136 & 100 & Cytoplasmic & 9.26 & $\mathrm{~J}$ \\
\hline $16-17$ & Mbov_0739 & \begin{tabular}{|l|} 
Putative \\
lipoprotein \\
(MbovP739) \\
\end{tabular} & gi|392051712| & 70.2 & 8.5 & 431 & 100 & Unknown & - & - \\
\hline 15 & Mbov_0789 & $\begin{array}{l}\text { Leucyl } \\
\text { aminopeptidase } \\
\text { (pepA) }\end{array}$ & gi|392051762| & 41.0 & 7.0 & 312 & 100 & Cytoplasmic & 9.64 & $E$ \\
\hline $1-2$ & Mbov_0797 & $\begin{array}{l}\text { Variable surface } \\
\text { lipoprotein } \mathrm{K} \\
(\text { VspK) } \\
\end{array}$ & gi|392051770| & 27.8 & 5.1 & 128 & 100 & Unknown & - & - \\
\hline 21 & Mbov_0839 & $\begin{array}{l}\text { LacI family } \\
\text { transcriptional } \\
\text { regulator }\end{array}$ & gi|392051809| & 37.2 & 9.1 & 124 & 100 & Cytoplasmic & 9.97 & K \\
\hline
\end{tabular}

*C.I. \%: Confidence Interval

**COG (Cluster of orthologous group) database functional classes: $(\mathrm{J})$ translation, ribosomal structure and biogenesis $(\mathrm{C})$ energy production and conversion, $(\mathrm{G})$ carbohydrate transport and metabolism, (E) amino acid transport and metabolism, (R) General function prediction only, (P) Inorganic ion transport and metabolism, (K) Transcription.

WCPs using 2-DE identified 639 well-resolved spots corresponding to $84 \%$ of the total number of coding sequences identified in the HB0801 genome (Figure 1A). Among those, 32 spots reacted with pooled sera from experimentally infected calves 35 days after infection (Figure 1B). No proteins reacted with the negative control sera. Mass spectrometry (MS raw dataset available at PRIDE repository-PXD003479) confirmed the presence of proteins in 21 spots (Figures 1A and 1B), corresponding to 16 different $M$. bovis proteins. Single spots identified 11 proteins, while five proteins were characterized by 2,4 , and 8 isoforms, suggesting post-translational modifications of these proteins (Table 1). Among these 16 antigenic proteins, 12 proteins belong to a broad range of functional categories, while 4 are hypothetical proteins with unknown functions (Table 1). Remarkably, only 7 proteins were predicted to be surface-exposed or membrane-associated: lipoproteins MbovP579 and P48-like, variable surface protein $\mathrm{K}$ (VspK), $\mathrm{F}_{0} \mathrm{~F}_{1}$ ATP synthase subunit beta (AtpD), phosphonate $\mathrm{ABC}$ transporter substrate-binding protein, 


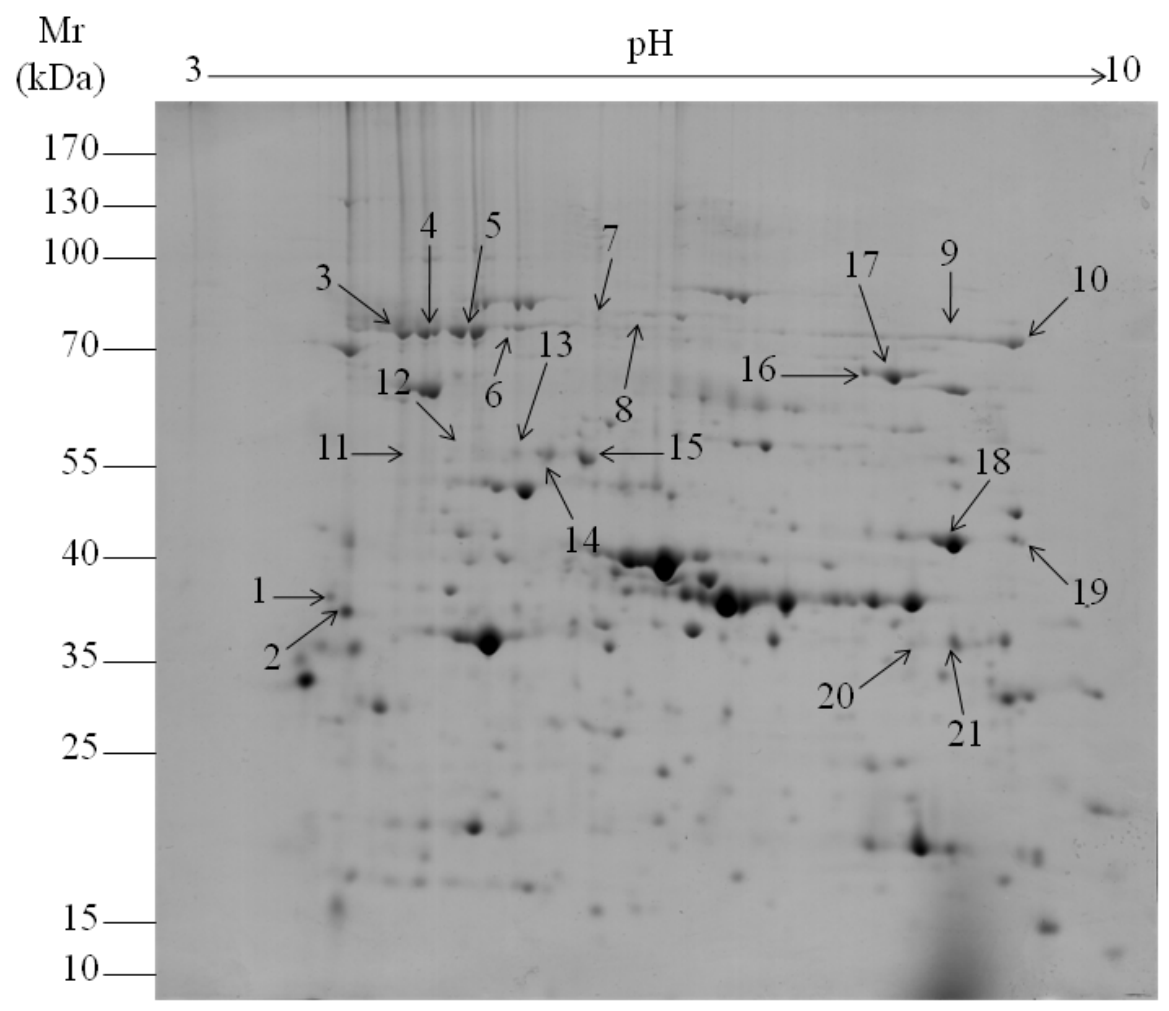

(A) 2-DE map of whole cell proteins of M. bovis HB0801

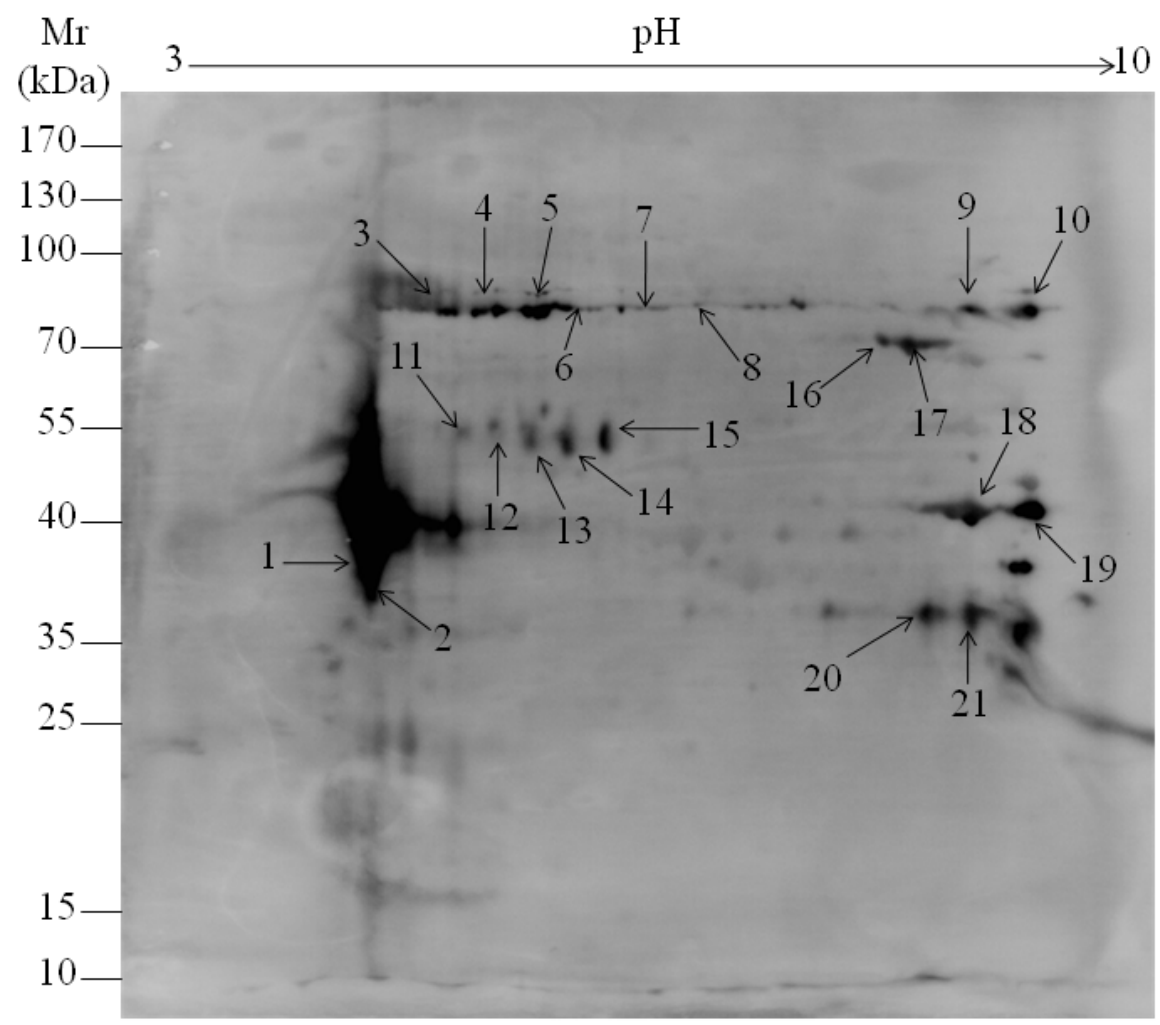

(B) Immunoblotting of whole cell proteins of M. bovis HB0801

Figure 1: 2D gel electrophoresis and western blot analysis of $\boldsymbol{M}$. bovis HB0801 whole cell proteins. A. Analysis of HB0801 whole cell proteins with 2-DE. Isoelectric points are indicated at the top and molecular weight markers in $\mathrm{kDa}$ on the left. B. Immunoblotting patterns of M. bovis HB0801 whole cell proteins obtained using a pool of sera derived from calves experimentally infected with HB0801. The 21 spots identified by MALDI-TOF MS are indicated on the 2D electrophoresis gel and the PVDF membrane. 
Table 2: Antigenic proteins in the $M$. bovis HB0801 TX114 membrane fraction

\begin{tabular}{|c|c|c|c|c|c|c|c|c|c|c|}
\hline $\begin{array}{l}\text { Spot } \\
\text { no. }\end{array}$ & Gene & Protein & NCBI ID & $\begin{array}{l}\text { Mw } \\
\text { (kDa) }\end{array}$ & pI & $\begin{array}{l}\text { Protein } \\
\text { Scores }\end{array}$ & \begin{tabular}{|l|} 
Protein \\
Score \\
C.I.\%* $\%$ \\
\end{tabular} & $\begin{array}{l}\text { PSORTb } \\
\text { Localization }\end{array}$ & $\begin{array}{l}\text { PSORTb } \\
\text { Probability }\end{array}$ & $\mathrm{COG}^{* *}$ \\
\hline 11 & Mbov_0016 & $\begin{array}{|ll|}\text { P48-like } & \text { surface } \\
\text { lipoprotein } & \\
\end{array}$ & gi|392051019| & 51.2 & 8.8 & 138 & 100 & \begin{tabular}{|l} 
Outer \\
Membrane
\end{tabular} & 9.52 & $\mathrm{R}$ \\
\hline 17 & Mbov_0038 & \begin{tabular}{|l|} 
putative \\
transmembrane \\
protein
\end{tabular} & gi|392051041| & 379.67 & 7.9 & 76 & 99.534 & $\begin{array}{l}\text { Outer } \\
\text { Membrane }\end{array}$ & 9.49 & - \\
\hline $6-9$ & Mbov_0111 & putative lipoprotein & gi|392051111| & 110.11 & 8.45 & 1,080 & 100 & Unknown & - & - \\
\hline 9 & Mbov_0115 & $\begin{array}{ll}\text { Oligopeptide } & \mathrm{ABC} \\
\text { transporter } & \mathrm{ATP} \\
\text { binding protein" }\end{array}$ & gi|392051115| & 94.0 & 8.9 & 57 & 62.107 & $\begin{array}{l}\text { Cytoplasmic } \\
\text { Membrane }\end{array}$ & 9.99 & $\mathrm{E}$ \\
\hline 17 & Mbov_0116 & $\begin{array}{l}\text { putative lipoprotein } \\
\text { (MbovP116) }\end{array}$ & gi|392051116| & 37.4 & 9.1 & 60 & 81.441 & Unknown & - & - \\
\hline $4-5$ & Mbov_0126 & $\begin{array}{l}\text { XAA-Pro } \\
\text { aminopeptidase } \\
\text { (pepP) }\end{array}$ & gi|392051126| & 39.8 & 6.05 & 460 & 100 & Cytoplasmic & 9.97 & $\mathrm{E}$ \\
\hline $16-17$ & Mbov_0130 & \begin{tabular}{|l} 
putative \\
transmembrane \\
protein \\
\end{tabular} & gi|392051130| & 26.3 & 9 & 64 & 91.319 & Unknown & - & $\mathrm{S}$ \\
\hline 15 & Mbov_0156 & putative lipoprotein & gi|392051154| & 37.1 & 8.85 & 201 & 100 & Unknown & - & - \\
\hline 11 & Mbov_0217 & putative lipoprotein & gi|392051211| & 53.5 & 9.13 & 342 & 100 & Unknown & - & - \\
\hline 13 & Mbov_0275 & $\begin{array}{l}\text { putative membrane } \\
\text { lipoprotein } \\
\text { (MbovP275) } \\
\end{array}$ & gi|392051268| & 40.1 & 8.51 & 118 & 100 & Unknown & - & - \\
\hline 1 & Mbov_0283 & Putative lipoprotein & gi|392051276| & 28.9 & 5.14 & 79 & 99.7 & Unknown & - & - \\
\hline 3 & Mbov_0299 & NADH oxidase & gi|392051292| & 49.9 & 6.09 & 76 & 99.4 & Cytoplasmic & 9.97 & $\mathrm{R}$ \\
\hline 11 & Mbov_0306 & $\begin{array}{l}\text { Phosphonate } \\
\text { ABC transporter } \\
\text { substrate-binding } \\
\text { protein }\end{array}$ & gi|392051299| & 51.5 & 9.04 & 374 & 100 & $\begin{array}{l}\text { Cytoplasmic } \\
\text { Membrane }\end{array}$ & 9.97 & $\mathrm{P}$ \\
\hline $4-5$ & Mbov_0312 & $\begin{array}{l}\text { Alcohol } \\
\text { dehydrogenase }\end{array}$ & gi|392051305| & 37.9 & 6.51 & 284 & 100 & Cytoplasmic & 9.97 & ER \\
\hline 12 & Mbov_0468 & putative lipoprotein & gi|392051449| & 65.5 & 8.35 & 255 & 100 & Unknown & - & - \\
\hline 7 & Mbov_0505 & putative lipoprotein & gi|392051486| & 90.4 & 7.64 & 411 & 100 & Unknown & - & - \\
\hline 17 & Mbov_0512 & hypothetical protein & gi|392051491| & 36.4 & 8.93 & 62 & 85.921 & Cytoplasmic & 8.96 & - \\
\hline 9 & Mbov_0515 & Putative lipoprotein & gi|392051494| & 97.5 & 8.81 & 58 & 71.255 & \begin{tabular}{|l} 
Outer \\
Membrane
\end{tabular} & 9.52 & - \\
\hline $7-9$ & Mbov_0517 & \begin{tabular}{|l} 
Putative \\
transmembrane \\
protein
\end{tabular} & gi|392051496| & 83.9 & 8.34 & 168 & 100 & $\begin{array}{l}\text { Outer } \\
\text { Membrane }\end{array}$ & 9.49 & - \\
\hline $10-17$ & Mbov_0579 & \begin{tabular}{|l} 
lipoprotein \\
(MbovP579)
\end{tabular} & gi|392051558| & 81.6 & 9.1 & 980 & 100 & Unknown & - & - \\
\hline 12 & Mbov_0580 & Nuclease & gi|392051559| & 32.9 & 6.92 & 154 & 100 & Extracellular & 9.97 & $\mathrm{~L}$ \\
\hline $\begin{array}{l}13- \\
14-15\end{array}$ & Mbov_0594 & $\begin{array}{ll}\text { cobalt/nickel } & \text { ABC } \\
\text { transporter } & \text { ATP- } \\
\text { binding protein } & \\
\end{array}$ & gi|392051573| & 35.3 & 6.69 & 155 & 100 & $\begin{array}{l}\text { Cytoplasmic } \\
\text { Membrane }\end{array}$ & 8.78 & $\mathrm{P}$ \\
\hline $16-17$ & Mbov_0693 & \begin{tabular}{|l|} 
putative \\
transmembrane \\
protein
\end{tabular} & gi|392051667| & 302.21 & 8.41 & 70 & 97.819 & Extracellular & 8.91 & - \\
\hline $7-8$ & Mbov_0739 & $\begin{array}{l}\text { Putative lipoprotein } \\
\text { (MbovP739) }\end{array}$ & gi|392051712| & 70.2 & 8.45 & 214 & 100 & Unknown & - & - \\
\hline 3 & Mbov_0789 & $\begin{array}{l}\text { Leucyl } \\
\text { aminopeptidase } \\
\text { (pepA) }\end{array}$ & gi|392051762| & 50.4 & 5.63 & 84 & 99.9 & Cytoplasmic & 9.64 & E \\
\hline \begin{tabular}{l|}
$13-$ \\
$14-$ \\
$15-16$
\end{tabular} & Mbov_0796 & $\begin{array}{l}\text { variable } \\
\text { lipoprotein }\end{array}$ & gi|392051769| & 31.5 & 9.01 & 350 & 100 & Unknown & - & \\
\hline 2 & Mbov_0797 & $\begin{array}{|ll|}\text { Variable } & \text { surface } \\
\text { lipoprotein } & \\
\end{array}$ & $\mid$ gi|392051770| & 34.6 & 5.02 & 324 & 100 & Unknown & - & - \\
\hline
\end{tabular}




\begin{tabular}{|l|l|l|l|l|l|l|l|l|l|l|}
\hline 15-16 & Mbov_0798 & $\begin{array}{l}\text { variable surface } \\
\text { lipoprotein }\end{array}$ & gi|392051771| & 39.8 & 9.06 & 105 & 100 & Unknown & - \\
\hline 3 & Mbov_0838 & Putative lipoprotein & gi|392051808 & 50.3 & 6.95 & 133 & 100 & Periplasmic & 9.83 & - \\
\hline
\end{tabular}

*C.I. \%: Confidence Interval

**COG (Cluster of orthologous group) database functional classes: (E) amino acid transport and metabolism, (R) General function prediction only, (P) Inorganic ion transport and metabolism, (L) Replication, recombination and repair

putative transmembrane protein, and a putative lipoprotein encoded by Mbov_0739(MbovP739). The remaining nine antigenic proteins were located in the cytoplasm according to the PSORTb database (Table 1).

\section{Antigens identified via membrane fraction analysis of $\mathrm{HB} 0801$}

The membrane proteome of $M$. bovis was first fractionated with Triton-X-114 (TX-114) and then subjected to 2-DE to better resolve basic and liposoluble transmembrane molecules and MAPs. Most of the TX-114 soluble fractions were resolved at the basic end of a 17 $\mathrm{cm}$ immobilized $\mathrm{pH}$ gradient (IPG) strip [pH 3-10 nonlinear (NL)] in the 2-DE map (Figure 2A). After Western blot analysis, protein spots that reacted strongly with antisera from experimentally infected calves 35 days after infection were excised from the 2-DE gel and subjected to MS. MS analysis (MS/MS raw dataset is available in the PRIDE repository - PXD003479) recognized the presence of proteins in 17 spots (Figure 2B), corresponding to 29 different $M$. bovis proteins. Sera from the negative control animals did not react with any of the $M$. bovis proteins. Eighteen proteins were recognized as single spots, 9 proteins were recognized in two spots, the variable surface lipoprotein [coding sequence (CDs) Mbov_0796] was identified in 4 isoforms, and cobalt/nickel $\mathrm{ABC}$ transporter ATP-binding protein (CDs Mbov_0594) was recognized in 3 spots (Table 2). Five of the 29 antigenic proteins were located in the cytoplasm, 10 were predicted to be MAPs, and the remaining 14 lipoproteins had unknown functions according to the PSORTb database (Table 2).

\section{Animal infection and immunization}

Increased titers of serum antibodies and gross lesion evaluation in postmortem examinations confirmed the success of animal infection experiments. Antibody levels were increased in the infected and immunized calves compared to negative control animals. Different lesions typical of infection, such as consolidation at the apical lung lobe and adhesions between the chest wall and the lung surface, were only observed in the infected group, and the mean pathological score of infected calves $(22.7 \pm 5.1)$ was higher than that of immunized $(5.3 \pm 3.1)$ and control $(3.3 \pm 1.5)$ calves $(p<0.01)$.

\section{Immunoblotting of recombinant membrane lipoproteins and cytoplasmic proteins}

The antigenic proteins identified by immunoproteiomics were analyzed in silico to select candidate proteins for further evaluation. Based on immunoinformatics analysis, 8 proteins (Table 3 ) were predicted to have a large number of T- and B-cell epitopes (Table S7-S10). These 8 highly-conserved antigenic $M$. bovis proteins (Table 3) were cloned and expressed in E. coli. The genes were first subjected to a site-directed mutagenesis (UGA to UGG) using overlapping PCR. Collectively, 25 site mutations were performed in the 8 genes (Table S1). The full genes were then cloned into pET-30a $(+)$ vectors and sequenced. Alignment assays confirmed the success of mutagenesis; the cloned genes matched the published sequence of HB0801 at the nucleotide level, except for the above modifications, and were inserted into the vector correctly. Expression of recombinant proteins was then induced with isopropylb-D-1-thiogalactopyranoside (IPTG, Invitrogen) and confirmed with SDS-PAGE (Figure 3A). After purification based on the his-tag, the 8 recombinant proteins were separated by SDS-PAGE, transferred to a membrane, and probed with antisera from both experimentally and naturally infected animals. rMbovP579 and recombinant MbovP739 (rMbovP739) were identified using serum antibodies from naturally infected animals, sera from calves 35 days after experimental infection with HB0801, and serum samples from calves immunized with $M$. bovis-150 35 days after immunization (Figure 3C). The other 2 lipoproteins and all 4 recombinant cytoplasmic proteins (Table 3 ) displayed weak signals in response to sera from all animal groups (Figure 3B).

\section{Characterization of the antigenicity of recombinant proteins with iELISA}

The in vivo expression and antigenicity of serum antibodies for $M$. bovis HB0801 WCPs and all 8 recombinant proteins (Table 3) were analyzed using antisera from both experimentally and naturally infected animals. In an iELISA based on the $M$. bovis WCPs, $40 \%$ $(8 / 20)$ of experimentally infected calves 14 days postinfection (dpi) and all infected calves 21 dpi were positive for antibody responses, whereas none of the vaccinated 


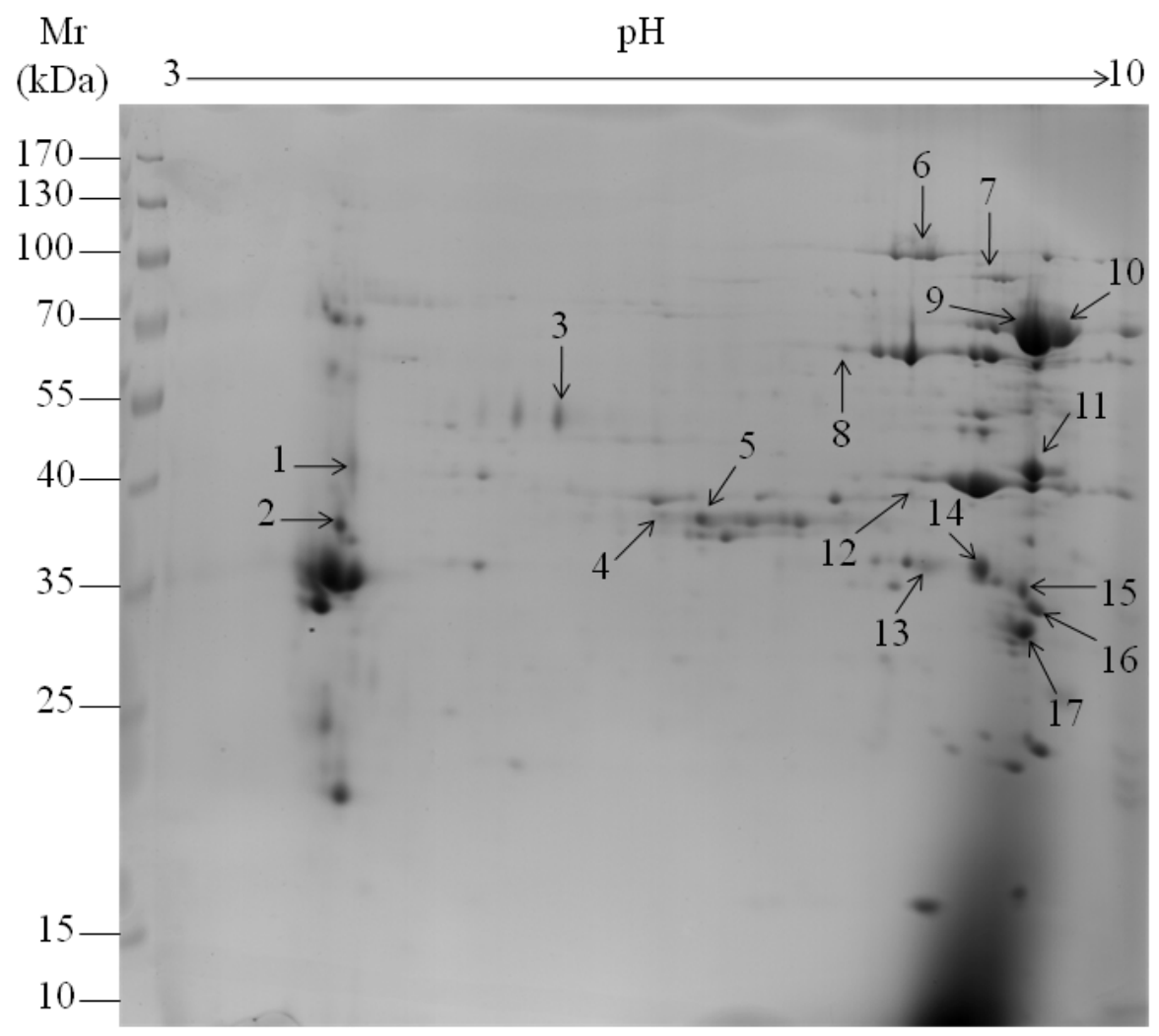

(A) 2-DE map of TX114 membrane fraction of M. bovis HB0801

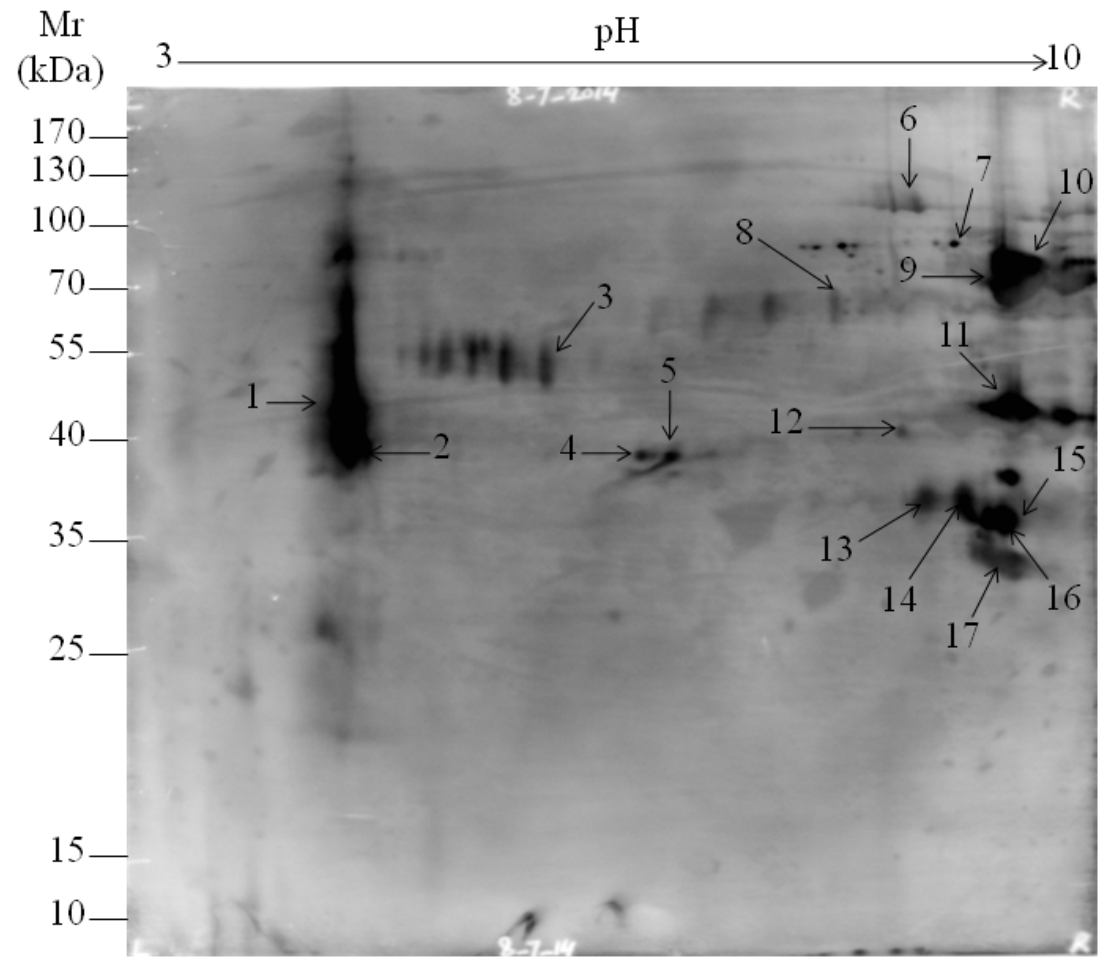

(B) Immunoblotting of TX114 membrane fraction of M. bovis HB0801

Figure 2: 2D gel electrophoresis and western blot analysis of $M$. bovis HB0801 TX114 membrane fractions. A. Resolution of the HB0801 TX114 membrane fraction using 2-DE. Isoelectric points, ranging from pH 3-10, are indicated at the top and molecular weight markers in $\mathrm{kDa}$ on the left. B. Antigenic pattern of the M. bovis HB0801 membrane fraction obtained using a serum pool from calves experimentally infected with HB0801. The 17 spots identified by MALDI-TOF MS are indicated on the 2-DE map and the PVDF membrane. 
Table 3: List of $M$. bovis $\mathrm{HB} 0801$ antigens expressed in $E$. coli

\begin{tabular}{|c|c|c|c|c|c|c|c|c|c|c|}
\hline \multirow{3}{*}{$\begin{array}{l}\text { Antigenic proteins } \\
\text { of } \\
\text { M.bovis HBo801 } \\
\text { with NCBI protein } \\
\text { ID }\end{array}$} & \multirow{3}{*}{$\begin{array}{l}\text { Mnemonic } \\
\text { in } \begin{array}{c}\text { M.bovis } \\
\text { HB0801 }\end{array}\end{array}$} & \multicolumn{9}{|c|}{ Homologs of HB0801 antigens in other sequenced $M$. bovis strains } \\
\hline & & \multicolumn{3}{|c|}{ M. bovis PG45 } & \multicolumn{3}{|c|}{ M. bovis Hubei-1 } & \multicolumn{3}{|c|}{ M. bovis CQ-W70 } \\
\hline & & Gene name & \begin{tabular}{|c|} 
Identity \\
$\%$
\end{tabular} & $\begin{array}{c}\text { NCBI protein } \\
\text { ID } \\
\end{array}$ & Gene name & $\begin{array}{c}\text { Identity } \\
\%\end{array}$ & \begin{tabular}{|l|}
$\begin{array}{l}\text { NCBI } \\
\text { protein ID }\end{array}$ \\
\end{tabular} & Gene name & $\begin{array}{c}\text { Identity } \\
\%\end{array}$ & \begin{tabular}{|l} 
NCBI \\
protein ID
\end{tabular} \\
\hline $\begin{array}{l}\text { Putative lipoprotein }{ }^{\mathrm{a}} \\
\text { (MbovP116) } \\
\text { (AFM51491.1) }\end{array}$ & Mbov_0116 & MBOVPG45_0117 & 94 & ADR25135.1 & MMB_0110 & 100 & AEI89824.1 & K668_00555 & 100 & AIA33703.1 \\
\hline $\begin{array}{l}\text { Putative membrane } \\
\text { lipoprotein }^{\mathrm{a}} \\
\text { (MbovP275) } \\
\text { (AFM51643.1) }\end{array}$ & Mbov_0275 & MBOVPG45_0584 & 96 & ADR24848.1 & MMB_0254 & 100 & AEI89968.1 & K668_01285 & 100 & AIA33843.1 \\
\hline $\begin{array}{l}\text { Lipoprotein } \\
(\text { MbovP579)a } \\
(\text { AFM51933.1) }\end{array}$ & Mbov_0579 & MBOVPG45_0311 & 92 & ADR25025.1 & MMB_0540 & 100 & AEI90254.1 & K668_02710 & 100 & AIA34118.1 \\
\hline $\begin{array}{l}\text { Putative lipoprotein } \\
\text { (MbovP739) } \\
\text { (AFM52087.1) }\end{array}$ & Mbov_0739 & MBOVPG45_0747 & 92 & ADR24729.1 & MMB_0704 & 100 & AEI90416.1 & K668_03510 & 100 & AIA34272.1 \\
\hline $\begin{array}{l}\text { Dihydrolipoamide } \\
\text { dehydrogenase } \\
\text { (DLD) } \\
\text { (AFM51481.1) }\end{array}$ & Mbov_0106 & MBOVPG45_0108 & 100 & ADR24676.1 & MMB_0100 & 100 & AEI89814.1 & K668_00505 & 100 & AIA33693.1 \\
\hline $\begin{array}{l}\text { XAA-Pro } \\
\text { aminopeptidase } \\
\text { (pepP) }^{\text {b }} \\
\text { (AFM51501.1) }\end{array}$ & Mbov_0126 & MBOVPG45_0127 & 99 & ADR24864.1 & MMB_0120 & 100 & AEI89834.1 & K668_00605 & 100 & AIA33713.1 \\
\hline $\begin{array}{l}\text { Transketolase (tkt) }{ }^{\mathrm{b}} \\
(\text { AFM51581.1) }\end{array}$ & Mbov_0212 & MBOVPG45_0650 & 99 & ADR25127.1 & MMB_0198 & 100 & AEI89912.1 & K668_01000 & 100 & AIA33788.1 \\
\hline $\begin{array}{l}\text { Leucyl } \\
\text { aminopeptidase } \\
\text { (pepA) b } \\
\text { (AFM52137.1) }\end{array}$ & Mbov_0789 & MBOVPG45_0802 & 100 & ADR25327.1 & MMB_0755 & 100 & AEI90467.1 & K668_03765 & 100 & AIA34323.1 \\
\hline
\end{tabular}

${ }^{\text {a }}$ Membrane-associated protein

${ }^{\mathrm{b}}$ Cytoplasmic protein

calves were positive until 21 days after immunization (Figures 4A and 4B). As expected, no antibody response against $M$. bovis WCPs was detected in the uninfected animals at any time point or in calves before infection or on the day of vaccination.

In contrast to the immunoblotting assays, iELISA demonstrated that all of the recombinant proteins generated antibody responses in both experimentally and naturally infected animals, indicating that recombinant protein antigenicity depended on their 3-dimensional structures in addition to primary structures.

Based on the intensity of antibody responses, the 4 recombinant membrane-associated lipoproteins (Table 3) together induced higher antibody responses than the 4 recombinant cytoplasmic proteins (recombinant dihydrolipoamide dehydrogenase (rDLD), XAA-Pro aminopeptidase (rpepP), transketolase (rTkt), and leucyl aminopeptidase (rpepA)). Remarkably, the IgG response to rMbovP579 was highest $(p<0.001)$, and rMbovP739 elicited the second-highest response $(p<0.05)$, in all animal groups compared to the other recombinant membrane lipoproteins and cytoplasmic proteins.

Serum antibodies were detected in 5\% (1/20) of calves $7 \mathrm{dpi}$ and in all infected animals $14 \mathrm{dpi}$ in the rMbovP579-based iELISA. While sera from $62.5 \%(5 / 8)$ of the vaccinated animals showed positive antibody responses in the rMbovP579-based iELISA 14 days after immunization, sera from all vaccinated animals showed positive responses after 35 days (Figures 4A and 4B). In the rMbovP739 assay, sera from $30 \%(6 / 20), 55 \%(11 / 20)$, and $100 \%$ of infected calves were positive 14,21 , and 35 dpi, respectively (Figures 4A and 4B), whereas antibody levels in immunized animals were lower than those in infected calves throughout the experiment, likely due to the lower dose administered to vaccinated animals. In assays based on recombinant lipoproteins encoded by CDs Mbov 0116 (rMbovP116) and CDs Mbov_0275 (rMbovP275), sera from 20\% (4/20) of the infected calves and $25 \%(2 / 8)$ of the vaccinated calves were positive 14 days after treatment; sera from all calves were positive by day 35 post-infection/vaccination, although antibody levels were relatively low (Figures 4A and 4B). Antisera responses to rDLD, rpepP, rTkt, and rpepA were low compared to responses to lipoproteins for both experimentally infected and immunized calves (Figures $4 \mathrm{~A}$ and $4 \mathrm{~B})$.

Among the recombinant lipoproteins, titers of antibodies against rMbovP579 and rMbovP739 were higher than those against the cytoplasmic proteins rDLD, rpepP, rTkt, and rpepA in the sera from naturally infected animals $(p<0.01)$. Meanwhile, the reactivity of rDLD, rpepP, rTkt, and rpepA with antisera was higher for naturally infected animals than for experimentally infected animals (Figure 4C). Additionally, the reaction of 
Table 4: Homologues of M. bovis HB0801 lipoprotein (MbovP579) in other mycoplasmas

\begin{tabular}{|c|c|c|c|c|}
\hline Pathogen & Mnemonics & Predicted gene function & \begin{tabular}{|l|} 
Query \\
coverage $(\%$ \\
of residue $)$
\end{tabular} & Identity $\%$ \\
\hline M. bovis PG45 & MBOVPG45_0311 & Membrane lipoprotein P81 & 98 & 92 \\
\hline M. bovis Hubei-1 & MMB_0540 & P80, lipoprotein & 100 & 100 \\
\hline M. bovis CQ-W70 & K668_02710 & Membrane lipoprotein P81 & 100 & 100 \\
\hline M.bovis NM 2012 & AAV31_02845 & Lipoprotein & 100 & 99 \\
\hline M. agalactiae $\mathrm{PG} 2$ & MAG5030 & P80, predicted lipoprotein & 99 & 66 \\
\hline M. agalactiae 14628 & MAGb_1420 & P80, predicted lipoprotein & 100 & 66 \\
\hline $\begin{array}{l}\text { M. mycoides subsp. mycoides SC } \\
\text { str. PG1 }\end{array}$ & MSC_0519 & Prolipoprotein B & 52 & 22 \\
\hline M. bovigenitalium 51080 & MBVG_0790 & Hypothetical protein & 97 & 27 \\
\hline M. mycoides subsp. capri PG3 & MMC_3250 & $\begin{array}{l}\text { Hypothetical protein, } \\
\text { predicted lipoprotein }\end{array}$ & 10 & 25 \\
\hline M. mycoides subsp. capri GM12 & MMCAP2_0459 & Glycerol ABC transporter & 52 & 23 \\
\hline $\begin{array}{l}\text { M. capricolum subsp. capricolum } \\
\text { ATCC } 27343\end{array}$ & MCAP_0451 & Putative lipoprotein & 10 & 28 \\
\hline M. ovipneumoniae Y98 & - & - & - & No similarity \\
\hline M. ovipneumoniae 14811 & MOVI_3720 & Hypothetical protein & 88 & 24 \\
\hline M. arginini ATCC 23838 & - & - & - & No similarity \\
\hline M. arginini 7264 & MARG_2010 & Hypothetical protein & 97 & 24 \\
\hline Mycoplasma hominis ATCC 27545 & MLBD4_00370 & $\begin{array}{l}\text { Putative polynucleotide- } \\
\text { binding lipoprotein }\end{array}$ & 96 & 25 \\
\hline
\end{tabular}

rMbovP579 with serum antibodies in naturally infected animals was higher than that of the other lipoproteins $(p<$ 0.01) (Figure 4C).

\section{Specificity of the highly antigenic lipoproteins}

The highly antigenic lipoproteins (MbovP579 and MbovP739) were analyzed further. Polyclonal antibodies against rMbovP579 and rMbovP739 were raised in $\mathrm{Balb} / \mathrm{c}$ mice to determine cross-reactivity with other common ruminant pathogens. Western blot analysis with these monospecific antibodies was used to identify the expression of these proteins in the M. bovis HB0801, attenuated M. bovis-150, and M. bovis typed (PG45) strains. No cross-reactivity was observed with WCPs from E.coli (BL21-DE 3 strain) or other mycoplasma species, including Mycoplasma mycoides subsp. capri (PG3), Mycoplasma ovipneumoniae (Y98), and Mycoplasma arginini. Moreover, while the rMbovP739 antibody displayed cross-reactivity with Mycoplasma agalactiae
(PG2) WCPs, no cross-reactivity was observed between the rMbovP579 antibody and other mycoplasma WCPs, demonstrating its high specificity for $M$. bovis (Figures 5A.2 and 5B.2). Amino acid sequence homologies for MbovP579 with other mycoplasmas are shown in Table 4. No IgGs reactive to both rMbovP579 and rMbovP739 were identified in reference antiserum against IBRV and BVDV with a WCPs-based iELISA.

\section{Performance of the rMbovP579-based iELISA}

Because of the specificity of MbovP579 and its conservation in all sequenced $M$. bovis strains (Table 3), including all other sequenced Chinese strains (unpublished data) (Table S2), an iELISA based on rMbovP579 was developed to confirm its value as a diagnostic biomarker. Optimal concentrations of the antigen and positive and negative sera were determined separately for the iELISA. The $\mathrm{OD}_{630}$ ratios of positive $(\mathrm{P})$ to negative sera $(\mathrm{N})$ were analysed. Based on a high $\mathrm{P} / \mathrm{N}$ ratio and $\mathrm{OD}_{630}$ values of 
approximately 1.00 for positive serum samples, the optimal antigen and serum dilutions were determined to be $250 \mathrm{ng} /$ well and 1:1600, respectively, with a $\mathrm{P} / \mathrm{N}=2.293$. The optimal secondary antibody dilution was 1:5000 (v/v), and the cut-off value $\left(\mathrm{OD}_{630}\right)$ was calculated as 0.442 by $\mathrm{ROC}$ analysis (Table S3). Compared to the gold standard test (M. bovis isolation and characterization), the diagnostic sensitivity of the rMbovP579-based iELISA was $90.2 \%$
(95\% CI: $83.7 \%, 94.3 \%$ ) and the specificity was $97.8 \%$ (95\% CI: $88.7 \%, 99.6 \%$ ) for sera from naturally infected and uninfected control animals (Figure 6 and Table S3, S4). For sera from experimentally infected and negative control calves, diagnostic sensitivity was $100 \%(20 / 20)$ and specificity was $100 \%(8 / 8)$ (Table S5). The estimated Kappa agreement value between the two methods was 0.7108 . Furthermore, the specificity of this iELISA was

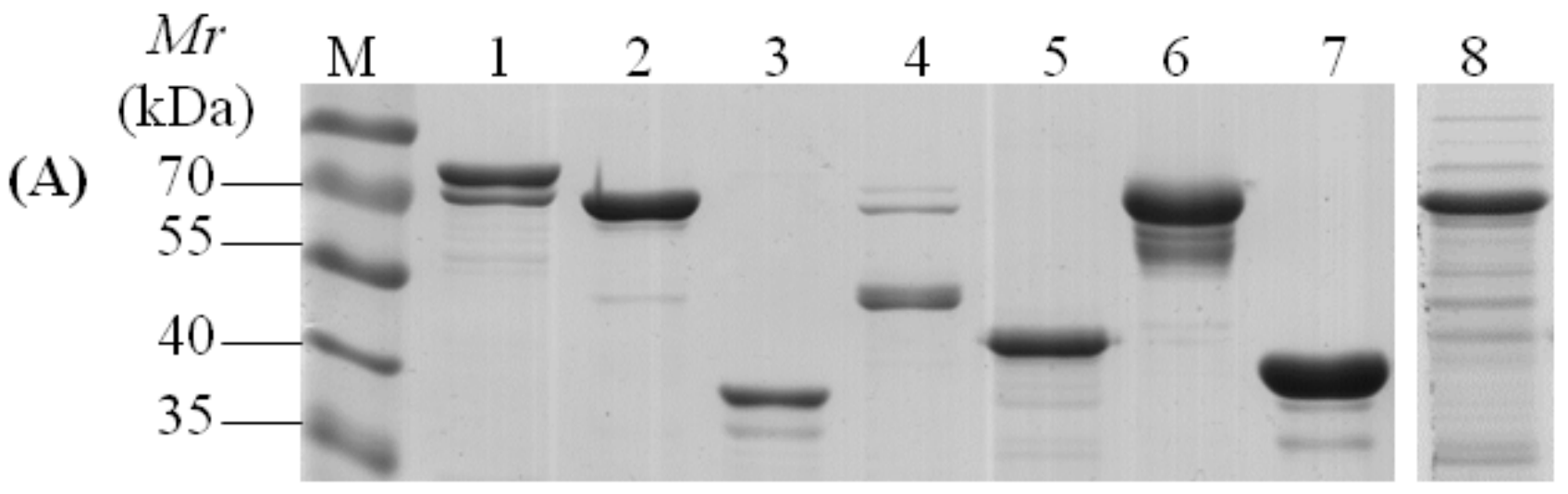

(B)
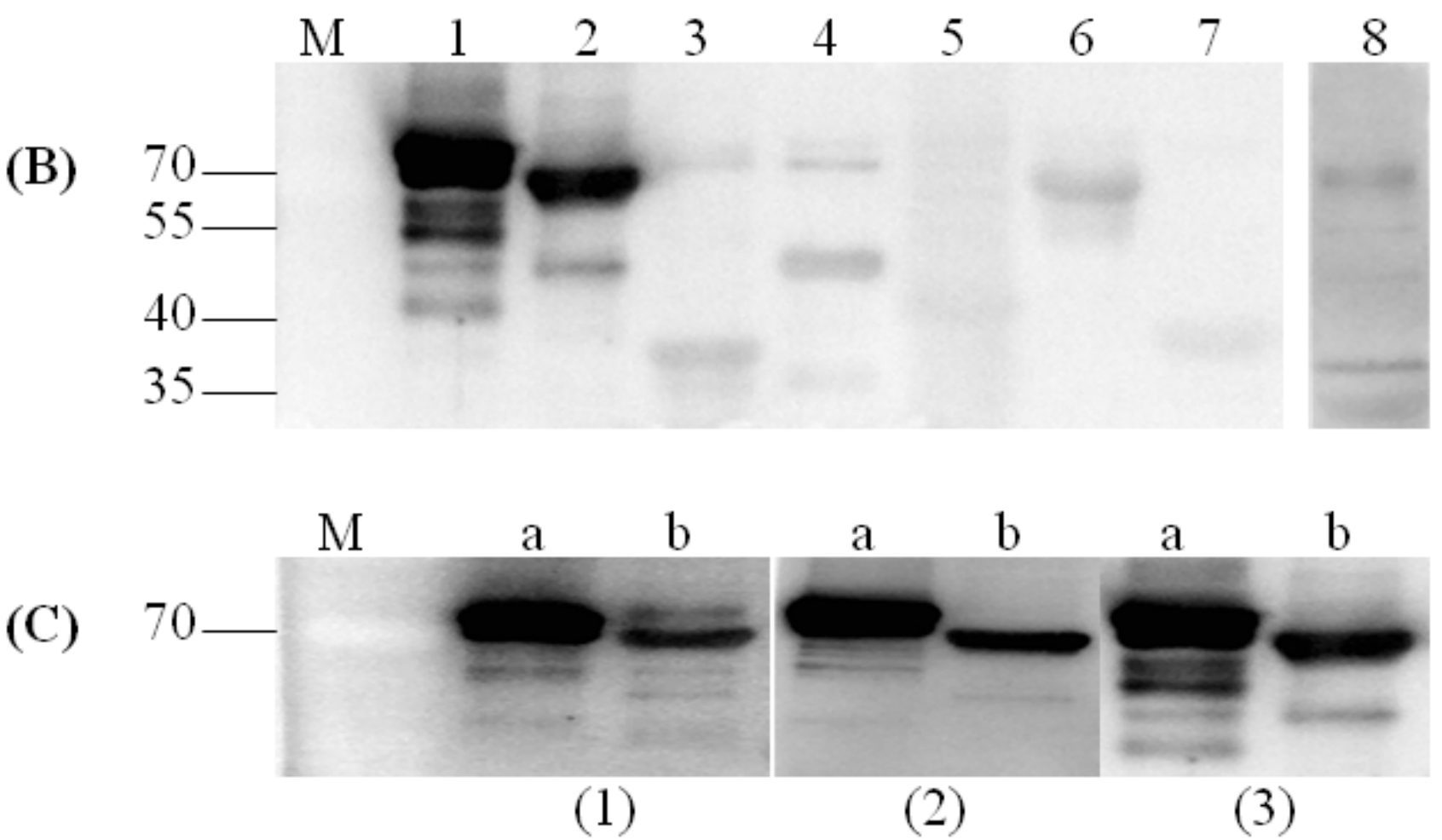

Figure 3: Representative SDS-PAGE and Western blotting assay of recombinant proteins. A. SDS-PAGE of recombinant proteins; B. Immune-reactivity of all recombinant proteins with antiserum samples from naturally infected cattle; C. Immunoblotting of recombinant lipoproteins (rMbovP579 and rMbovP739) with antisera from experimental calves 35 dpi (1), experimental calves 35 days post-vaccination (2), and naturally infected cattle (3). Protein markers are indicated by " $\mathrm{M}$ " with the respective molecular weights in kDa on the left. Lanes 1-8 in A. and B. indicate proteins rMbovP579, rMbovP739, rMbovP116, rpepA, rpepP, rDLD, rMbovP275, and rTkt, respectively; lanes A. and B. in C. indicate rMbovP579 and rMbovP739, respectively. Serum samples from each group of cattle were diluted at 1:100. 
determined by testing the antisera against common cattle pathogens, including infectious bovine rhinotracheitis virus (IBRV) and bovine viral diarrhoea virus (BVDV). $\mathrm{OD}_{630}$ values were less than $0.40(0.207 \pm 0.042)$ for all serum samples, confirming the specificity of this iELISA.

\section{Comparison of efficiency between rMovP579- based iELISA and a commercial kit}

Parallel serum samples from 20 experimentally infected calves and 123 naturally infected cattle were tested using the rMbovP579-based iELISA and a commercially available kit. All experimentally infected animals (20/20) were identified as positive with the rMbovP579-based iELISA, whereas only 65\% (13/20) were positive according to the commercial kit. In addition, the sensitivity of the iELISA for these samples was $90.2 \%$ (95\% CI: $83.7 \%, 94.3 \%$ ) compared to only $31.7 \%$ for the

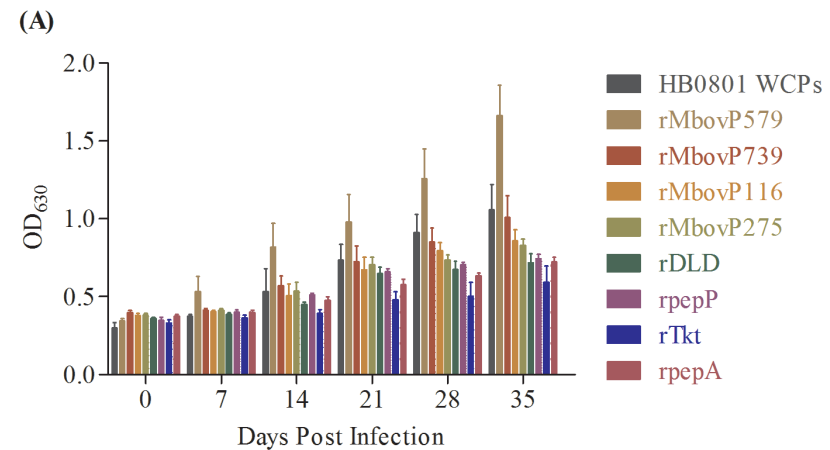

commercial kit. The serum samples identified as negative for the M. bovis-specific IgG by the iELISA were also found to be negative with the commercial kit (Table S6). These results demonstrate that the rMbovP579-based iELISA was more sensitive than the commercial kit ( $p<$ $0.01)$. To determine the analytic sensitivity of both assays, 93 positive and 25 negative sera were individually twofold serially diluted $(1: 100-1: 102,400)$ and evaluated separately using both methods. The iELISA detected the $\mathrm{IgG}$ at dilutions as high as 1:51,200; however, the maximum detectable dilution for the commercial kit was 1:6400 with an 8-fold decrease. Thus, the analytic sensitivity of the rMbovP579-based iELISA was higher than that of the commercial kit $(p<0.05)$.

\section{Unique characteristics of MbovP579}

The specificity of MbovP579 was further confirmed by a gene (Mbov_0579) sequence assay. Alignments of the

(B)

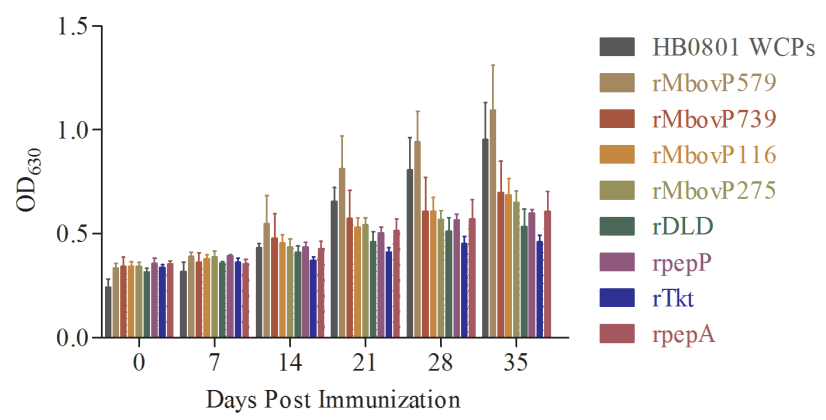

(C)

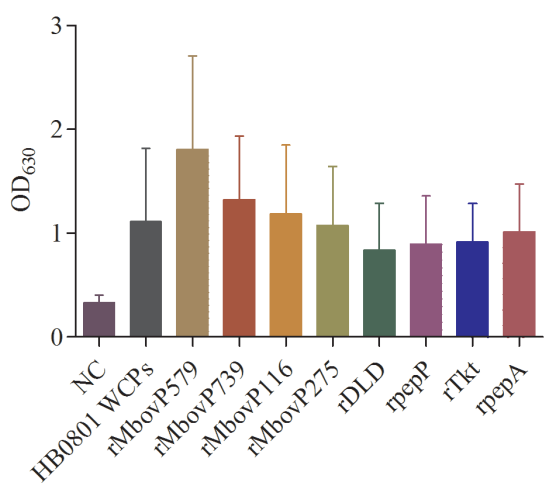

Figure 4: Reactions of $M$. bovis $\mathrm{HB} 0801$ whole cell proteins and recombinant proteins to different antisera measured with iELISA. $M$. bovis HB0801 whole cell proteins (WCPs) and all recombinant proteins (rMbovP579, rMbovP739, rMbovP116, rMbovP275, rDLD, rpepP, rTkt, and rpepA) were characterized in vivo with antiserum samples from each experimentally infected calf 0-35 dpi A., antisera from each experimentally immunized calf 0-35 days post-vaccination B., and serum samples from each animal naturally infected with $M$. bovis $\mathbf{C}$. The Y-axis indicates mean \pm SD iELISA $\mathrm{OD}_{630}$ values of serum antibodies from infected, vaccinated, and uninfected (NC) animals against WCPs and all recombinant proteins. Serum antibodies to proteins were diluted as follows: WCPs 1:400, rMbovP579 1:1600, rMbovP739 1:200, and rMbovP116, rMbovP275, rDLD, rpepP, rTkt, and rpepA 1:100. 
M. bovis HB0801 Mbov_0579 gene with other sequenced M. bovis strains, including PG45 (CP002188.1), Hubei-1 (CP002513.1), CQ-W70 (CP005933.1), and NM2012 (CP011348.1), showed 95\%, 100\%, 100\%, 99\% gene-level similarities, respectively, while the nucleotide sequence was 74\% similar to a homologous gene in Mycoplama agalactiae. The lack of genes orthologous to Mbov_0579 in other pathogens further supported its specificity as a diagnostic antigen.

Using SignalP4.1 and PRED-LIPO, MbovP579 was identified as a secreted peptide with reliability scores of 0.591 and 0.96 , respectively. The MbovP579 signal peptide sequence, MSKKNKLMIGLSSTAIPLLAAVSA|KC, was a 24-amino-acid fragment at the $\mathrm{N}$-terminal and contained a putative peptidase I cleavage site between the alanine and lysine residues at positions 24 and 25, resulting in the release of a mature protein with lysine as the first residue. Interestingly, MbovP579 secretion was also predicted by a nonclassical secretory pathway with a high SecP score of 0.84 .

In addition, the predicted template structure for MbovP579 according to ElliPro was an ADPribosyltransferase toxin known as Clostridium difficile toxin subunit "a" (CDTa) (PDB ID- 2WN4) (Figure S-1A). CDTa is believed to have catalytic activity. Alignment of the MbovP579 and CDTa amino acid sequences revealed similarity of approximately $31 \%$ for amino acids residues 591-674 at the MbovP579 C-terminus (Figure S-1B).

\section{DISCUSSION}

Mycoplasma bovis infection is a major threat to the worldwide cattle industry. It was initially detected in China in 2008 as an emerging pathogen in feedlots and continues to cause economic losses. Due to the lack of an efficient preventive strategy, the control of $M$. bovis is largely reliant on management practices and early chemotherapy. Therefore, researchers have focused on diagnostic laboratory tests for the early detection and subsequent treatment of diseases related to $M$. bovis infection in beef and dairy herds. Generally, serological assays are highly reliable because antibodies remain detectable for several months in infected animals, even after antibiotic treatments [20, 23]. Additionally, because antigenic variation is prevalent in both virulent and attenuated $M$. bovis strains $[4,11]$, more conserved,
$M r$

$(\mathrm{kDa})$

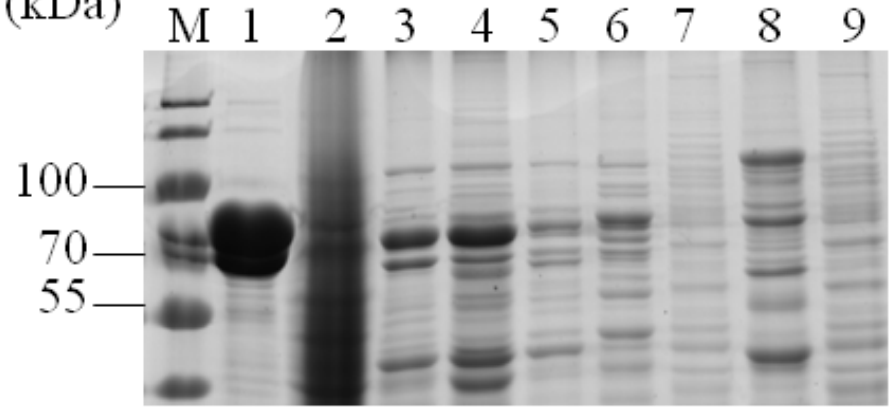

(B1)

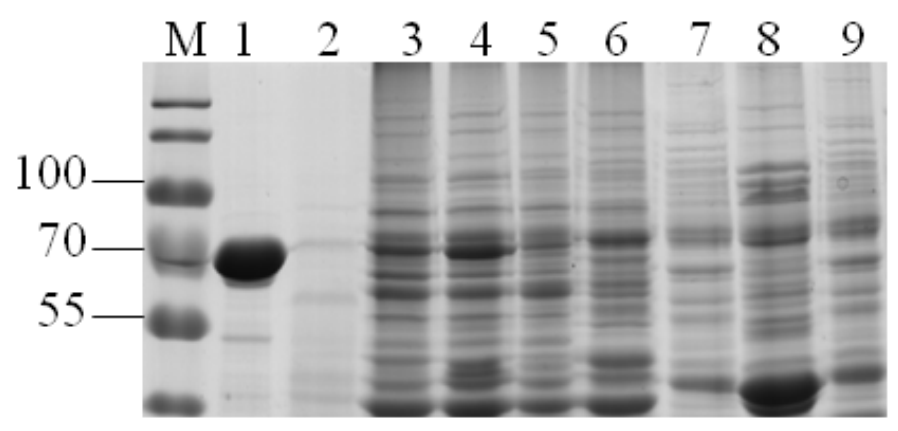

(A2)

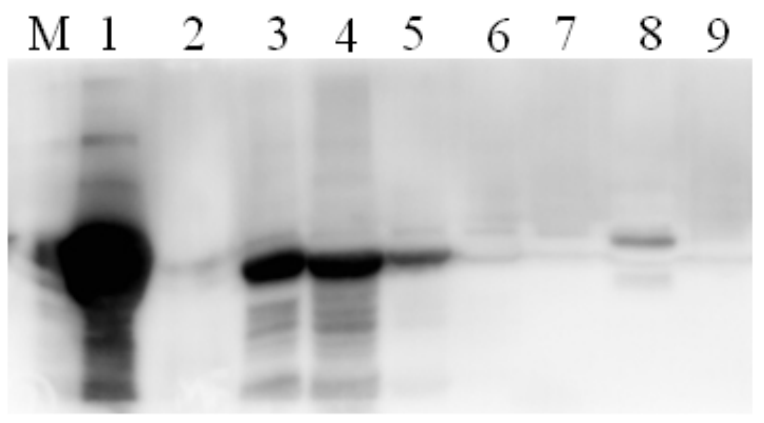

(B2)

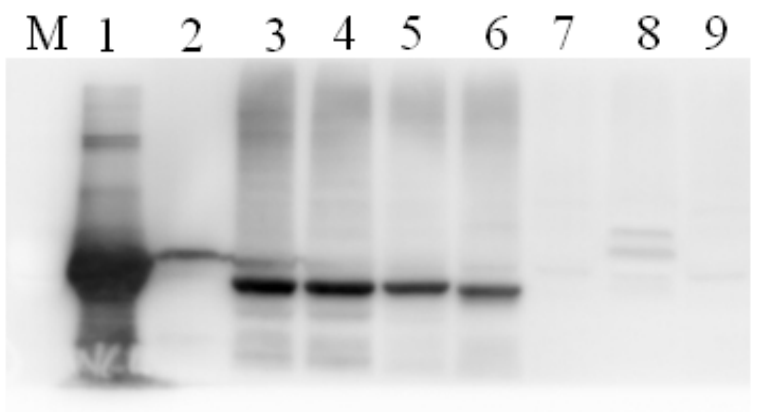

Figure 5: MbovP579 and MbovP739 expression in $M$. bovis strains and other mycoplasmas. A1. SDS-PAGE of rMbovP579 $(81.6 \mathrm{kDa})$ and WCPs of mycoplasmas; A2. Western blot analysis with mouse anti-rMbovP579 polyclonal antibodies; B1. SDS-PAGE of rMbovP739 (70.2 kDa) and WCPs of mycoplasma strains; B2. Western blot analysis with mouse anti-rMbovP739 polyclonal antibodies. Protein markers are indicated by "M" with the respective molecular weights in kDa on the left. Lanes 1-9: rMbovP579/rMbovP739, E. coli

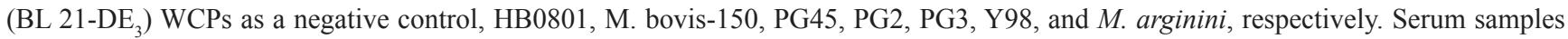
were diluted at 1:100. 
sensitive, and specific antigens for $M$. bovis are urgently required to help develop reliable serodiagnostic methods for the early detection of infection and assessments of vaccine efficacy. Here, we identified several previously unreported antigenic $M$. bovis proteins and characterized a promising candidate diagnostic biomarker protein.

\section{Global antigenic profile of $M$. bovis identified using immunoproteomics}

During the last decade, immunoproteomics approaches, such as 2-DE combined with Western blotting and MS, have increasingly been used to detect antigens in many pathogens, including several mycoplasma species $[20,24,25,26,34,35]$. In this study, we analyzed $M$. bovis WCPs together with membrane fractions using immunoproteomics for the first time to comprehensively identify antigenic $M$. bovis proteins. Collectively, 39 antigenic proteins were identified, 6 of which were present in both the WCPs and membrane fractions. Thirty-two novel antigenic proteins, most of which were MAPs, were identified (Table 1, 2). The discovery of 23 additional antigens in the $M$. bovis membrane fractions confirmed the importance of membrane proteins in pathogen antigenicity.

\section{Confirmation of previously identified antigens}

Five of the antigenic proteins in our protein list, VspK, PDHB, DLD, P48-like lipoprotein, and MilA (encoded by CDS Mbov 0693 in HB0801), have previously been identified in other $M$. bovis strains. In addition, the antigenicity of DLD has been reported in many other ruminant mycoplasma species, including several members of the mycoides phylogenetic cluster $[20,24,25,35]$.

Three other important antigenic proteins in HB0801 identified here are AtpD, TktA, and the elongation factor Tu (Ef-Tu). Although they have been identified as antigens in other mycoplasma species, these proteins have not been identified in previous studies of $M$. bovis. AtpD has been used in a serological assay for the diagnosis of $M$. pneumonia infections in humans [36]. AtpD immunoreactivity has also been reported in $M$. mycoides subsp. mycoides SC, Brucella abortus, and Brucella melitensis [24, 37, 38]. Similarly, the antigenic properties of TktA and Ef-Tu have been reported in several mycoplasma species [24, 25, 35, 39]. However, AtpD might have lower specificity as a diagnostic marker for M. bovis due to its $83 \%$ similarity with the amino acid sequence of AtpD in M. mycoides subsp. mycoides SC and M. mycoides subsp. capri (NCBI database).

Three of the proteins we identified, P48-like lipoprotein, MilA, and PDHB, have been evaluated for serodiagnosis of $M$. bovis [5, 17, 20]; however, assays based on P48 and rPDHB have relatively low sensitivities of $56.3 \%$ and $51 \%$, respectively, in sera from naturally infected animals. The sensitivity and specificity of the recombinant MilA-based assay for clinical bovine serum samples have not yet been determined [17].

VspK is a phase variable antigen encoded by a cluster of hypervariable surface exposed lipoproteins, called the Vsp locus, in M. bovis [15]. The antigenicand virulence-related properties of $V_{s p s}$ and pMB67 (a

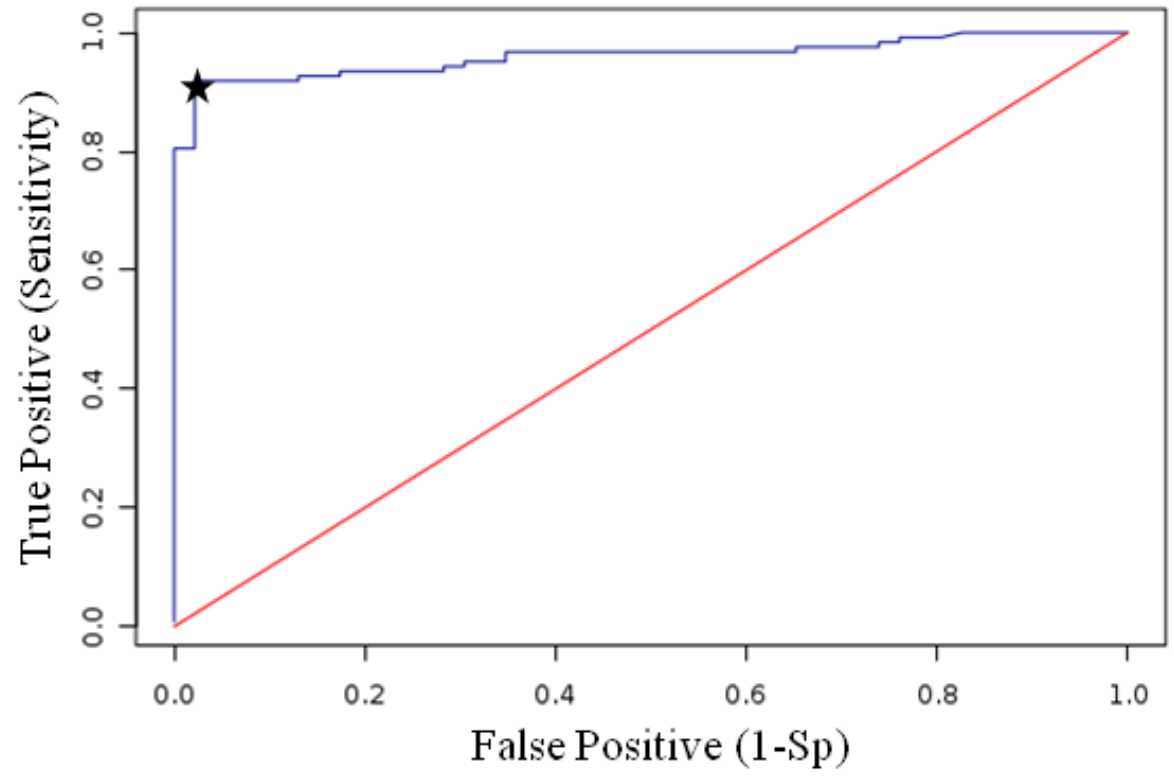

Figure 6: ROC analysis of the rMbovP579-based iELISA compared to gold standard tests. Based on the ROC curve, 0.442 was determined as the iELISA cut-off value with a sensitivity of $90.2 \%$ and specificity of $97.8 \%$. 
$V s p$-unrelated variable membrane protein) have been described elsewhere $[12,13]$. Although $V s p \mathrm{~K}$ exhibited a strong reactivity with animal sera, immune response to $V s p$ s is non-protective due to variations in expression, conformation, and antigenicity [17, 40]. Thus, highly variable antigenic proteins, such as $V_{s p \mathrm{~K}}$ and pMB67, are not the best options for serodiagnostic assays or subunit vaccine development.

Other $M$. bovis antigens identified in previous studies, such as pMB67, heat shock protein 60, and GAPDH, were not detected in this study. This might be due to differences in methods and antisera used for antigen identification. For instance, pMB67 was detected to be antigenic in $M$. bovis total proteins by radiolabeling, SDSPAGE, and immunostaining using monoclonal antibodies [12], while the antigenicity of recombinant Hsp60 [18] and GAPDH [19] were identified by conventional serological methods with antisera from naturally infected animals. Here, proteomic technology and antisera from experimentally infected animals were used to identify antigenic proteins, which were then confirmed using antisera from naturally infected animals. It is possible that these two types of antisera may identify different antigenic profiles for $M$. bovis strains due to differential infection doses and courses in experimental versus natural infection.

\section{The novel rMbovP579-based iELISA is the most sensitive and specific method for detecting $M$. bovis-specific antibodies}

Among the 8 antigenic recombinant proteins discovered in this study, MbovP579 was the most sensitive, specific, and conserved antigen. An rMbovP579-based iELISA was thus established. This iELISA could detect seroconversion $7 \mathrm{dpi}$ and by day 14 after vaccination. To the best of our best knowledge, this iELISA, which was far more sensitive than a commercially available kit, may currently be the most sensitive and specific method for $M$. bovis antigen detection.

Previous assays often failed to differentiate antibody responses against $M$. bovis from those against $M$. agalactiae infection because these mycoplasma species are closely phylogenetically related. Surprisingly, the rMbovP579-based iELISA was able to differentiate the antibody responses induced by these two species. Nucleotide alignment revealed that the Mbov_0579 gene is not only conserved among $M$. bovis strains, but is also unique to $M$. bovis compared to other pathogens. For example, the similarity of this gene at the nucleotide level is only $74 \%$ between $M$. bovis and $M$. agalactiae, and no orthologues were found in other pathogens.

According to published data [4], the function of the MbovP579 lipoprotein is unknown. We performed a bioinformatics assay to further characterize this protein. It was predicted to be a secretory lipoprotein since it has a 24-amino-acid signal sequence at the N-terminal [41, 42, 43]. More interestingly, the predicted MbovP579 template structure was ADP- ribosyltransferase CDTa (PDB ID- 2WN4). The functional domains of ADPribosyltransferase have recently been identified in the community-acquired respiratory distress syndrome (CARDS) toxin of Mycoplasma pneumonia [44]. In this study, using the ElliPro modeling tool, we predicted that MbovP579 might be an ADP-ribosyltransferase toxin, although the amino acid similarity between the MbovP579 C-terminus from amino acids 591-674 and CDTa is only $31 \%$. Whether it functions as protein toxin remains to be established in future studies.

In conclusion, we identified and characterized MbovP579 as a promising novel diagnostic biomarker for $M$. bovis using immunoproteomics. The highly sensitive and specific rMbovP579-based iELISA would likely aid in the early detection of $M$. bovis infection.

\section{MATERIALS AND METHODS}

\section{Ethical statement about animal experiments}

Experimental protocols involving animals were approved by the Hubei Province Science and Technology Department, which is responsible for ethics in animal experiments in Hubei, China (permit no. SYXK(ER) 2010-0029), in accordance with China Regulations for the Administration of Affairs Concerning Experimental Animals (1988) and the Hubei Regulations for the Administration of Affairs Concerning Experimental Animals (2005). Animal experiments were performed under the supervision of the Ethical Committee for Experimental Animals of Huazhong Agricultural University, Wuhan, China.

\section{Strain and culture conditions}

The virulent Chinese M. bovis HB0801 strain (CCTCC \# M2010040), isolated from a lung lesion in a calf with pneumonia [45], and the attenuated vaccine strain M. bovis-150 (CCTCC \# M2011102), generated by our laboratory in vitro [3], were used in this study. Bacteria were cultured as previously described [2]. Stock cultures were stored as aliquots at $-80^{\circ} \mathrm{C}$. The Mycoplasma bovis PG45, Mycoplasma agalactia (PG2), Mycoplasma mycoides subsp. capri (PG3), Mycoplasma ovipneumoniae (Y98), and Mycoplasma arginini, and E.coli strains were stored in our laboratory and cultured as previously described [46]. IBRV (\#AV20) and BVDV (\#AV69) were purchased from the China Institute of Veterinary Drug Control and were grown according to the provided instructions. 


\section{Extraction of whole cell proteins (WCPs)}

HB0801 WCPs were extracted as previously described [47], with some minor modifications. Briefly, HB0801 cell pellets were re-suspended in PBS containing protease inhibitors (Roche, USA), and disrupted with a French press (Thermo, USA) at $20,000 \mathrm{lb} / \mathrm{in}^{2}$. After centrifugation $\left(15,400 \mathrm{~g}, 20 \mathrm{~min}, 4^{\circ} \mathrm{C}\right)$, supernatant was treated with $15 \%$ TCA for $1 \mathrm{~h}$ at $-20^{\circ} \mathrm{C}$. Following centrifugation, the protein pellet was washed with cold acetone to remove TCA and re-suspended in lysis buffer [8 $\mathrm{M}$ urea, $2 \mathrm{M}$ thiourea, 4\% CHAPS, 2\% Amidosulfobetaine-14 (ASB-14), 60 mM DTT, $40 \mathrm{mM}$ Tris- $\mathrm{HCl} \mathrm{pH}$ 8.8]. All the reagents were purchased from Sigma (USA). Protein concentration was measured with the 2D Quant Kit (GE healthcare, Sweden). Solubilized proteins were used immediately or stored at $-80^{\circ} \mathrm{C}$ until use.

\section{Preparation of membrane-associated proteins (MAPs)}

M. bovis MAPs were fractionated using TX-114 as previously described [48] with minor modifications. In brief, M. bovis HB0801 pellet was re-suspended in PBS containing 4\% TX-114 (Sigma) and 1 mM PMSF (Sigma) and kept for $3-5 \mathrm{~h}$ at $4{ }^{\circ} \mathrm{C}$ after scraping. Un-lysed cells and debris were precipitated and removed by centrifugation for $15 \mathrm{~min}$ at $15,400 \mathrm{~g}$. The supernatant was then incubated for $20 \mathrm{~min}$ at $37^{\circ} \mathrm{C}$ and then centrifuged for $5 \mathrm{~min}$ at $7500 \mathrm{~g}$ to separate the two phases. The upper aqueous phase was discarded and the lower detergent phase was reconstituted to the original volume with $1 \mathrm{mM}$ PMSF in PBS for washing. After washing, the proteins in the detergent phase were re-established in $100 \%$ methanol and incubated overnight at $-80^{\circ} \mathrm{C}$. After centrifugation for $20 \mathrm{~min}$ at $15,400 \mathrm{~g}$, the proteins were re-suspended in lysis buffer. Protein concentration was measured with a 2D quant kit (GE Healthcare, Sweden).

\section{Preparation of experimental anti-sera}

Thirty-six clinically healthy 5-6 month old local calves were confirmed to be free of M. bovis as described previously [49]. These animals were randomly allocated among 3 groups. The 20 animals in the infected group were inoculated intratracheally with HB0801 for three consecutive days at a dose of $10^{9} \mathrm{cfu} / \mathrm{calf}$. The 8 animals in the vaccinated group were vaccinated with the attenuated M. bovis-150 strain in a single intranasal dose of $10^{8}$ cfu/calf. The control group included 8 calves that were exposed to sterile PPLO media as a negative control. Serum samples were collected from all animals on days $0,7,14,21,28$, and 35 after infection/immunization. The animals were euthanized and necropsied 40 days after infection/vaccination for gross lesion evaluation using a previously described scoring system [3]. The antibody titers of serum samples were measured by iELISA as described previously [49]. Serum samples collected on day 0 before infection or immunization were used as negative controls.

\section{Serum samples from the naturally infected and uninfected animals}

Serum samples were collected from 46 uninfected calves and 123 calves infected with M. bovis, as determined by isolations conducted in our laboratory, from various cattle feedlots in China. Disease was diagnosed as described previously [49]. All serum samples were stored at $-80^{\circ} \mathrm{C}$ for further analysis.

\section{Two dimensional gel electrophoresis (2-DE)}

2-DE with IPG strips was conducted as previously reported [50] with some modifications. In brief, $17 \mathrm{~cm}$ IPG strips (pH 3-10 NL) were actively rehydrated at 50V with either M. bovis whole cell lysate or the TX-114 soluble fraction (380 $\mu \mathrm{g} / \mathrm{strip})$ in rehydration solution [7 M Urea, 2 M Thiourea, 2.5\% w/v CHAPS, 2\% w/v ASB-14, $40 \mathrm{mM}$ Tris-HCl, $\mathrm{pH} 8.8,65 \mathrm{mM}$ DTT, 0.5\% IPG buffer $\mathrm{pH} 3-10$ (Bio-Rad), $0.002 \% \mathrm{w} / \mathrm{v}$ bromophenol blue (Sigma, USA)] for $16 \mathrm{~h}$ at $20^{\circ} \mathrm{C}$ using a Protean IEF cell (Bio-Rad). Isoelectric focusing (IEF) was executed using the following program: $150 \mathrm{~V}$ for $3 \mathrm{~h}, 300 \mathrm{~V}$ for $3 \mathrm{~h}, 1000 \mathrm{~V}$ (gradient) for $6 \mathrm{~h}, 10000 \mathrm{~V}$ (gradient) for $3 \mathrm{~h}$, and $10000 \mathrm{~V}$ for 60 $\mathrm{kVhs}$ (kilovolt hours). After focusing, the strips were equilibrated with $2 \%$ DTT and $4 \%$ iodoacetamide (Sigma, USA), respectively, in $10 \mathrm{~mL}$ equilibration solution [6 M urea, $50 \mathrm{mM}$ Tris- $\mathrm{HCl} \mathrm{pH} \mathrm{8.8,30 \%} \mathrm{glycerol,} \mathrm{2 \%} \mathrm{SDS]}$ for $15 \mathrm{~min}$. The second separation was performed on $10 \%$ gels (SDS-PAGE) at $12^{\circ} \mathrm{C}$ for $3 \mathrm{~h}$ at $50 \mathrm{~V}$ followed by $12 \mathrm{~h}$ at $100 \mathrm{~V}$ using the Protean II xi multi-cell with 2-D conversion kit (Bio-Rad, USA). All experiments were run in triplicate. Proteins were stained on the first gel with $0.15 \%$ Coomassie brilliant blue R-250 (CBB R-250- Bio-Rad, USA), scanned with a GS-800 Calibrated Densitometer (Bio-Rad), and evaluated with the PD Quest Basic 8.0 program (Bio-Rad, USA). Proteins from other gels were subjected to an immunoblotting assay.

\section{Immunoblotting assay}

Western blot analysis of the proteins separated by 2-DE was performed as described previously [47] with some modifications. Briefly, proteins separated on 2-DE gels were electro-blotted onto PVDF membranes, blocked with $5 \%$ dried skimmed milk powder, and probed for $1 \mathrm{~h}$ at 
room temperature with pooled sera (1:500) collected from all experimentally infected calves 35 days after infection. Pooled sera collected on day 0 before infection were used as negative controls. Immunoblots were established with horseradish peroxidase (HRP)-conjugated goat anti-bovine IgG (Pierce, USA, 1:5000) for $1 \mathrm{~h}$, developed with super signal west femto chemiluminescent substrate (Pierce, USA), and visualized on the Chemiluminescence \& Fluorescence DNr Bio-imaging system (DNr, Israel).

\section{Matrix-assisted laser desorption/ionization time of flight (MALDI-TOF) mass spectrometry (MS)}

Antigenic proteins were removed from the gel and digested with trypsin. In brief, gels were first treated with $200-400 \mu \mathrm{L}$ of destaining solution $\left(100 \mathrm{mM} \mathrm{NH}_{4} \mathrm{HCO}_{3}\right.$ in $30 \% \mathrm{ACN}$ ) and lyophilized. Peptide extraction was performed three times with $60 \% \mathrm{ACN} / 0.1 \%$ TFA, and proteins were freeze dried after gel digestion in $5 \mu \mathrm{L}$ (2.5$10 \mathrm{ng} / \mu \mathrm{L}$ ) of trypsin at $37^{\circ} \mathrm{C}$ overnight. Dry samples were reconstituted in $20 \% \mathrm{ACN}(2 \mu \mathrm{L})$ and loaded on a $384-$ well Opti-TOF $(123 \mathrm{~mm} \times 81 \mathrm{~mm})$ stainless steel plate. The samples in the plate were then overlaid with $0.5 \mu \mathrm{L}$ CHCA in $50 \%$ ACN and $0.1 \%$ TFA. Mass spectrometry and $\mathrm{MS} / \mathrm{MS}$ data for the recognition of proteins were generated with a MALDI-TOF appliance (4800 plus proteomics analyzer, Applied Biosystems). The conditions were fixed using the 4000 Series Explorer software (Applied Biosystems). The MS ranges were documented in reflector mode at masses ranging from 800 to $4000 \mathrm{Da}$ with a focal mass of 2000 Da. A CalMix 5 standard was used to adjust the MS equipment (ABI 4700 Calibration Mixture). For a single core MS spectrum, 25 subspectra with 125 shots per subspectrum were collected using a random search pattern. For MS standardization, trypsin autolysis peaks $([\mathrm{M}+\mathrm{H}]+842.5100$ and 2,211.1046) were used as internal controls, and up to 10 of the strongest ion signals were chosen as precursors for MS/MS analysis, excluding the autolysis peaks of trypsin and matrix ion signals. In MS/MS positive ion mode, for a single core MS spectrum, 50 subspectra with 50 shots per subspectrum were taken with a random search pattern. Collision energy was $2 \mathrm{kV}$, collision gas was air, and default calibration was set with Glu1-Fibrino-peptide B ( $[\mathrm{M}+\mathrm{H}]+1,570.6696)$ spotted onto the Cal 7 positions of the MALDI target. Collective peptide mass fingerprinting (PMF) and MS/ MS inquiries were accomplished by the MASCOT search engine 2.2 (Matrix Science, Ltd., U.S.) in the GPSExplorer Software 3.6 (Applied Biosystems) from the NCBI database (Taxonomy: NCBI_Bacteria 5910423, 12/3/2010,NCBI_Mycoplasma 198866, 22/5/2014). The search criteria used in this study were trypsin specificity, one missed cleavage, carbamidomethylation of cysteines as fixed modifications, and oxidation of methionine residues as dynamic modifications. The peptide mass tolerance was set to $\pm 100 \mathrm{ppm}$ and the MS/MS fragment tolerance was set to $\pm 0.4 \mathrm{Da}$. A GPS Explorer protein confidence interval $\geq 95 \%$ (protein score C.I. \%) was used for further validation. The identified protein sequences were obtained from NCBI. Upon comparison to a cluster of orthologous groups (COGs) database using RPS-BLAST, functional classification of proteins was determined, and subcellular localization of identified proteins was predicted with the PSORTb database.

\section{Prediction of T- and B-cell epitopes in M. bovis antigenic proteins}

The amino acid sequences of antigenic $M$. bovis HB0801 proteins were subjected to T- and B- cell epitope prediction with tools cited in the Immune Epitope Database and Analysis Resource (IEDB-AR), a database of experimentally identified B- and T-cell epitopes (http:// tools.immuneepitope.org/mhci/), as described previously [28]. Modeling of 3D structure templates was performed with the SWISS-MODEL Workspace (http://swissmodel. expasy.org) and ElliPro (http://tools.immuneepitope.org/ $\underline{\text { tools) }}[51,52]$. Templates were acquired for $M$. bovis proteins by modeling their sequences in the FASTA format. To predict B-cell conformational epitopes, a modeled template of each protein was submitted to Ellipro using conditions similar to those reported previously for cattle pathogens [28].

\section{Site-directed mutagenesis, cloning, expression, and purification of $\mathrm{HB} 0801$ antigens}

Eight highly conserved antigenic proteins (Table 3), with large numbers of predicted T- and B-cells epitopes, were selected for subsequent analysis. The complete genes of these M. bovis proteins were amplified by overlap extension PCR for the site-directed mutagenesis using the primers in Table S1 and pfu DNA polymerase (Thermo, USA) to avoid the translational hurdle of differences in UGA codon usage between mycoplasmas and $E$. coli. The complete genes, with single nucleotide changes in the UGA codon (UGA $\rightarrow$ UGG), were cloned into pET-30a $(+)$ vectors. The constructs were confirmed by nucleotide sequencing (Sangon Company, China). Recombinant plasmids were transformed into $E$. coli BL21 (DE3) (Novagen, USA) competent cells for expression. A single clone of each recombinant plasmid was inoculated in 5 $\mathrm{mL}$ of Luria-Bertani (LB) medium containing $120 \mu \mathrm{g} / \mathrm{mL}$ kanamycin and incubated in a shaker $\left(37^{\circ} \mathrm{C}, 250 \mathrm{rpm}\right)$ until the $\mathrm{OD}_{600}$ reached 0.6. Recombinant protein expression was induced with a $0.4 \mathrm{mM}$ final concentration of IPTG, and the culture was allowed to grow at $30^{\circ} \mathrm{C}$ for $4 \mathrm{~h}$. After extraction and washing with PBS, the recombinant proteins were purified by nickel affinity chromatography (GE Healthcare, Sweden). Protein concentrations were 
measured by the BCA method (Thermo, USA) and verified with $12 \%$ SDS-PAGE.

\section{Characterization of promising $M$. bovis antigens}

The 8 purified recombinant proteins $(2 \mu \mathrm{g})$ were separated by $12 \%$ SDS-PAGE and blotted onto a PVDF membrane. After blocking, recombinant proteins were probed with serum samples collected from the following groups of animals: (a) M. bovis HB0801-infected calves 35 days after infection; (b) M. bovis-150-immunized calves 35 days post-vaccination; and (c) naturally infected calves.

In addition, the eight recombinant antigenic proteins (Table 3) and M. bovis WCPs were characterized with iELISAs. iELISA conditions were optimized for $M$. bovis WCPs and each recombinant protein as described elsewhere [49]. In brief, 96-well microtitre plates were coated overnight at $4^{\circ} \mathrm{C}$ with $125 \mathrm{ng}$ of WCPs and 250 ng of each purified recombinant protein diluted in $100 \mu \mathrm{L}$ sodium carbonate buffer ( $\mathrm{pH}$ 9.6) and washed with PBS containing $0.05 \%$ Tween 20 (PBST). After blocking, the plates were probed for $1 \mathrm{~h}$ at $37^{\circ} \mathrm{C}$ with sera at various dilutions collected from the experimental and naturally infected groups of calves described above. After washing with PBST, plates were incubated for $1 \mathrm{~h}$ at $37^{\circ} \mathrm{C}$ with goat anti-bovine IgG-HRP (1:5000) (Southern Biotech Co. USA) and washed with PBST followed by the addition of tetramethylbenzidine (TMB) $/ \mathrm{H}_{2} \mathrm{O}_{2}$ (Wuhan Keqian Biological Co., Ltd, China) as a substrate. The reaction was stopped after $10 \mathrm{~min}$ and OD values were obtained with a microtiter plate reader (BioTek, USA) at $630 \mathrm{~nm}$ $\left(\mathrm{OD}_{630}\right)$. Cut-off $\mathrm{OD}_{630}$ values were determined for WCP and recombinant protein-based iELISAs using serum samples from the 46 uninfected animals. Mean $\mathrm{OD}_{630}$ values and standard deviations were calculated, and cutoff values were set at mean $+2 \mathrm{SD}$.

\section{Production of mouse polyclonal antibodies against MAPs}

Ten female BALB/c mice (5 weeks old) were purchased from the China Hubei Provincial Center for Disease Control and Prevention to produce polyclonal antibodies against rMbovP579 and rMbovP739. Mice were divided into three groups $(4$ mice in each protein group and 2 mice in the negative control group). Mice in each protein group were immunized subcutaneously with $100 \mu \mathrm{g}$ in $200 \mu \mathrm{L}$ of purified recombinant protein mixed with an equal volume of Freund's complete adjuvant (Sigma, USA). Two subsequent boosters, each with the same amounts of protein and Freund's incomplete adjuvant (Sigma, USA), were administered at intervals of 2 weeks. The serum titer of the polyclonal antibodies was evaluated after each immunization. When titers increased significantly, the mice were euthanized; antiserum to each protein was collected and stored at $-20^{\circ} \mathrm{C}$ for further use. Sera from each inoculated mouse on day 0 and serum samples from uninfected mice were used as negative controls.

\section{Specificity of the two lipoproteins}

In the Western blot analysis, the specificities of M. bovis MbovP579 and MbovP739 in WCPs from the following organisms, including related mycoplasma species, were determined using the mouse anti-rMbovP579 and anti-rMovP739 polyclonal antibodies: 1) M. bovis HB0801;2) attenuated vaccine strain $M$. bovis-150; 3) $M$. bovis PG45 (ATCC 25523); 4) Mycoplasma agalactiae PG2; 5) Mycoplasma mycoides subsp. capri (PG3); 6) Mycoplasma ovipneumoniae (Y98); 7) Mycoplasma arginini; and 8) E.coli strain $\mathrm{BL} 21-\mathrm{DE}_{3}$ as a negative control. The protein concentration of each sample was measured using the BCA method (Thermo, USA). The specificities of MbovP579 and MbovP739 were also determined by iELISA using reference antiserum against common cattle pathogens, including IBRV and BVDV. Each protein sample $(25 \mu \mathrm{g})$ was resolved on a $12 \%$ SDSPAGE gel and blotted onto a PVDF membrane at $15 \mathrm{~V}$ for 45 min using a trans-blot semi-dry transfer cell (BioRad). Homology of the MbovP579 amino acid sequence with other mycoplasma sequences was analyzed using the NCBI database (Table 4). All pathogens described above were identified using specific PCR before these experiments.

\section{Prediction of MbovP579 secretion}

In silico analysis was used to predict whether MbovP579 was a secretory or non-secretory protein. Both classical and non-classical secretion were included. Prediction of classical secretion and identification of the presence of signal peptides were conducted using SignalP 4.1 (http://www.cbs.dtu.dk/services/SignalP) and PREDLIPO (http://bioinformatics.biol.uoa.gr/PRED-LIPO/) as reported previously [41, 42]. SecretomeP 2.0 (http:// www.cbs.dtu.dk/services/SecretomeP) was used to predict nonclassical secretion [43]. The predictions were executed at a default cutoff value of 0.5 . Predicted scores of 0.5 and above were considered indicative of secretion.

\section{Establishment of rMbovP579-based iELISA}

An iELISA was established as described previously [49]. Briefly, 96-well ELISA plates were coated with rMbovP579 serially diluted two-fold from a concentration 
of $2 \mu \mathrm{g}$ to $0.97 \mathrm{ng} / \mathrm{well}$ and incubated at $4^{\circ} \mathrm{C}$ overnight. Sera were added at two-fold serial dilutions of 1:25, 1:50, $1: 100,1: 200,1: 400,1: 800$, and 1:1600 (v/v). Commercial goat anti-bovine IgG-HRP secondary antibody was diluted to $1: 3000,1: 5000$, and $1: 8000(\mathrm{v} / \mathrm{v}) . \mathrm{TMB} / \mathrm{H}_{2} \mathrm{O}_{2}$ was added as substrate to the wells and incubated for $10 \mathrm{~min}$, and the $\mathrm{OD}_{630}$ values were measured with a microplate reader. To compare the performance of iELISA and standard tests, serum samples from the 20 experimentally infected calves, the 8 uninfected experimental calves, and the 123 naturally infected and 46 uninfected animals from various feedlots were analyzed using this iELISA; sensitivity and specificity were determined based on rMbovP579 and Kappa agreement. The cut-off value was defined as the value where both sensitivity and specificity were highest in receiver operating characteristic (ROC) analysis. Additionally, antisera against IBRV and BVDV purchased from the China Institute of Veterinary Drug Control were evaluated with the iELISA to confirm its specificity.

\section{Comparison of the rMbovP579-based iELISA and a commercial Kit}

Serum samples from 123 naturally infected animals and 20 experimentally infected animals were separately subjected to the rMbovP579-based iELISA and the commercial kit (BioX, Belgium) under standardized conditions, and the sensitivity of the two assays, as well as the degree of agreement, were determined. Additionally, the analytic sensitivities of the rMbovP579-based iELISA and commercial kit were individually evaluated with 93 positive serum samples excluding samples with $\mathrm{OD}_{630}$ values $\geq 2.2 \pm 0.2$ or $\leq 0.8 \pm 0.2$. The sera were two-fold serially diluted from $1: 100$ to $1: 102,400$ and tested in parallel using both methods. The highest dilution for which the assay returned a positive result indicated the analytic sensitivity, while the proportion of true positives (determined by M.bovis isolation, the gold standard method) detected indicated the diagnostic sensitivity.

\section{Data availability}

The MS/MS proteomics data have been deposited in the ProteomeXchange Consortium (http://proteomecentral. proteomexchange.org) via the PRIDE [53] partner repository with the dataset identifier PXD003479.

\section{Statistical analysis}

The Kappa agreement coefficient between the assays, cut-off values, and sensitivity and specificity based on ROC analysis were determined online using EpiTools . The differences in analytic sensitivity between the rMovP579-based iELISA and commercial kit were analyzed using the Chi-square or Fisher's exact test. p values below 0.05 were considered statistically significant; $* \mathrm{p}<0.05, * * \mathrm{p}<0.01$.

\section{CONFLICTS OF INTEREST}

The authors declare no conflicts of interest.

\section{GRANT SUPPORT}

The work was supported by Special funds for Agroscientific research in the public interest from the People's Republic of China (No. 201003060), the National Natural Science Foundation (No. 31272587), and funds from the China Agriculture Research System (CARS No. 38).

\section{REFERENCES}

1. Nicholas RAJ and Ayling RD. Mycoplasma bovis: disease, diagnosis, and control. Res Vet Sci. 2003; 74: 105-112

2. Mustafa R, Qi J, Ba X, Chen Y, Hu C, Liu X, Tu L, Peng Q, Chen H, and Guo A. In vitro Quinolones Susceptibility Analysis of Chinese Mycoplasma bovis Isolates and their Phylogenetic Scenarios based upon QRDRs of DNA Topoisomerases Revealing a Unique Transition in ParC. Pak Vet J. 2013; 33: 364-369

3. Zhang R, Han X, Chen Y, Mustafa R, Qi J, Chen X, Hu C, Chen H, and Guo A. Attenuated Mycoplasma bovis strains provide protection against virulent infection in calves. Vaccine. 2014; 32: 3107-3114

4. Qi J, Guo A, Cui P, Chen Y, Mustafa R, Ba X, Hu C, Bai Z, Chen X, Shi L, and Chen H. Comparative Geno-Plasticity Analysis of Mycoplasma bovis HB0801 (Chinese Isolate). Plos One. 2012; 7.

5. Fu P, Sun Z, Zhang Y, Yu Z, Zhang H, Su D, Jiang F, and $\mathrm{Wu}$ W. Development of a direct competitive ELISA for the detection of Mycoplasma bovis infection based on a monoclonal antibody of P48 protein. BMC Vet Res. 2014; 10.

6. Gerchman I, Levisohn S, Mikula I, and Lysnyansky I. In vitro antimicrobial susceptibility of Mycoplasma bovis isolated in Israel from local and imported cattle. Vet Microbiol. 2009; 137: 268-275.

7. Ayling RD, Rosales RS, Barden G, and Gosney FL. Changes in antimicrobial susceptibility of Mycoplasma bovis isolates from Great Britain. Vet Rec. 2014; 175.

8. Gautier-Bouchardon AV, Ferre S, Le Grand D, Paoli A, Gay E, and Poumarat F. Overall Decrease in the Susceptibility of Mycoplasma bovis to Antimicrobials over the Past 30 Years in France. Plos One. 2014; 9. 
9. Lerner U, Amram E, Ayling RD, Mikula I, Gerchman I, Harrus S, Teff D, Yogev D, and Lysnyansky I. Acquired resistance to the 16-membered macrolides tylosin and tilmicosin by Mycoplasma bovis. Vet Microbiol. 2014; 168: 365-371

10. Sulyok KM, Kreizinger Z, Fekete L, Hrivnak V, Magyar T, Janosi S, Schweitzer N, Turcsanyi I, Makrai L, Erdelyi $\mathrm{K}$, and Gyuranecz M. Antibiotic susceptibility profiles of Mycoplasma bovis strains isolated from cattle in Hungary, Central Europe. BMC Vet Res. 2014; 10.

11. Thomas A, Leprince P, Dizier I, Ball H, Gevaert K, Van Damme J, Mainil J, and Linden A. Identification by twodimensional electrophoresis of a new adhesin expressed by a low-passaged strain of Mycoplasma bovis. Res Microbiol. 2005; 156: 713-718.

12. Behrens A, Poumarat F, Le Grand D, Heller M, and Rosengarten R. A newly identified immunodominant membrane protein (pMB67) involved in Mycoplasma bovis surface antigenic variation. Microbiology. 1996; 142 (9): 2463-2470.

13. Sachse K, Helbig JH, Lysnyansky I, Grajetzki C, Mueller W, Jacobs E, and Yogev D. Epitope mapping of immunogenic and adhesive structures in repetitive domains of Mycoplasma bovis variable surface lipoproteins. Infect Immun. 2000; 68: 680-687.

14. Sachse K, Grajetzki C, Rosengarten R, Hänel I, Heller $\mathrm{M}$, Pfützner $\mathrm{H}$. Mechanisms and factors involved in Mycoplasma bovis adhesion to host cells. Zentralbl Bakteriol. 1996; 284(1):80-92.

15. Citti C, Nouvel LX, and Baranowski E. Phase and antigenic variation in mycoplasmas. Future Microbiol. 2010; 5: 10731085.

16. Robino P, Alberti A, Pittau M, Chessa B, Miciletta M, Nebbia P, Le Grand D, and Rosati S. Genetic and antigenic characterization of the surface lipoprotein P48 of Mycoplasma bovis. Vet Microbiol. 2005; 109: 201-209.

17. Wawegama NK, Browning GF, Kanci A, Marenda MS, and Markham PF. Development of a Recombinant ProteinBased Enzyme-Linked Immunosorbent Assay for Diagnosis of Mycoplasma bovis Infection in Cattle. Clin Vaccine Immunol. 2014; 21: 196-202.

18. Scherm B, Gerlach GF, and Runge M. Analysis of heat shock protein 60 encoding genes of mycoplasmas and investigations concerning their role in immunity and infection. Vet Microbiol. 2002; 89: 141-150.

19. Perez-Casal J and Prysliak T. Detection of antibodies against the Mycoplasma bovis glyceraldehyde-3-phosphate dehydrogenase protein in beef cattle. Microb Pathog. 2007; 43: 189-197.

20. Sun Z, Fu P, Wei K, Zhang H, Zhang Y, Xu J, Jiang F, Liu $\mathrm{X}, \mathrm{Xu} \mathrm{W}$, and $\mathrm{Wu} \mathrm{W}$. Identification of Novel Immunogenic Proteins from Mycoplasma bovis and Establishment of an Indirect ELISA Based on Recombinant E1 Beta Subunit of the Pyruvate Dehydrogenase Complex. Plos One. 2014; 9.
21. Prysliak T, Van der Merwe J, and Perez-Casal J. Vaccination with recombinant Mycoplasma bovis GAPDH results in a strong humoral immune response but does not protect feedlot cattle from an experimental challenge with Mycoplasma bovis. Microb Pathog. 2013; 55: 1-8.

22. Hermeyer K, Buchenau I, Thomasmeyer A, Baum B, Spergser J, Rosengarten R, and Hewicker-Trautwein M. Chronic pneumonia in calves after experimental infection with Mycoplasma bovis strain 1067: Characterization of lung pathology, persistence of variable surface protein antigens and local immune response. Acta Vet. Scand. 2012; 54

23. Adamu JY, Wawegama NK, Browning GF, and Markham PF. Membrane proteins of Mycoplasma bovis and their role in pathogenesis. Res Vet Sci. 2013; 95: 321-325.

24. Jores J, Meens J, Buettner FFR, Linz B, Naessens J, and Gerlach GF. Analysis of the immunoproteome of Mycoplasma mycoides subsp mycoides small colony type reveals immunogenic homologues to other known virulence traits in related Mycoplasma species. Vet Immunol Immunopathol. 2009; 131: 238-245.

25. Pinto PM, Chemale G, De Castro LA., Costa APM, Kich JD, Vainstein MH, Zaha A, and Ferreira HB. Proteomic survey of the pathogenic Mycoplasma hyopneumoniae strain 7448 and identification of novel post-translationally modified and antigenic proteins. Vet Microbiol. 2007; 121: 83-93.

26 Zhao P, He Y, Chu YF, Gao PC, Zhang X, Zhang NZ, Zhao HY, Zhang KS, and Lu ZX. Identification of Novel Immunogenic Proteins in Mycoplasma capricolum subsp capripneumoniae Strain M1601. J Vet Med Sci. 2012; 74: 1109-1115.

27. Churchward CP, Rosales RS, Gielbert A, Dominguez $\mathrm{M}$, Nicholas RAJ, and Ayling RD. Immunoproteomic characterisation of Mycoplasma mycoides subsp capri by mass spectrometry analysis of two- dimensional electrophoresis spots and western blot. J Pharm Pharmacol. 2015; 67: 364-371.

28. Gurung RB, Purdie AC, Begg DJ, and Whittington RJ. In Silico Identification of Epitopes in Mycobacterium avium subsp paratuberculosis Proteins That Were Upregulated under Stress Conditions. ClinVaccine Immunol. 2012; 19: 855-864.

29. Gurung RB, Purdie AC, Begg DJ, and Whittington RJ. In silico screened Mycobacterium avium subsp paratuberculosis (MAP) recombinant proteins upregulated under stress conditions are immunogenic in sheep. Vet Immunol Immunopathol. 2012; 149: 186-196.

30. Mustafa AS and Shaban FA. ProPred analysis and experimental evaluation of promiscuous T-cell epitopes of three major secreted antigens of Mycobacterium tuberculosis. Tuberculosis. 2006; 86: 115-124.

31. Sardinas G, Climent Y, Rodriguez Y, Gonzalez S, Garcia D, Cobas K, Caballero E, Perez Y, Brookes C, Taylor S, Gorringe A, Delgado M, Pajon R, and Yero D. Assessment 
of vaccine potential of the Neisseria-specific protein NMB0938. Vaccine. 2009; 27: 6910-6917.

32. Vani J, Shaila MS, Chandra NR, and Nayak R. A combined immuno-informatics and structure-based modeling approach for prediction of $\mathrm{T}$ cell epitopes of secretory proteins of Mycobacterium tuberculosis. Microbes Infect. 2006; 8: 738-746.

33. Guerfali FZ, Ben-Abdallah H, Sghaier RM, Ben-Aissa K, Mkannez G, Attia H, and Laouini D. An in silico immunological approach for prediction of $\mathrm{CD} 8+\mathrm{T}$ cell epitopes of Leishmania major proteins in susceptible $\mathrm{BALB} / \mathrm{c}$ and resistant $\mathrm{C} 57 \mathrm{BL} / 6$ murine models of infection. Infect Genet Evol. 2009; 9: 344-350.

34. Cacciotto C, Addis MF, Pagnozzi D, Chessa B, Coradduzza E, Carcangiu L, Uzzau S, Alberti A, and Pittau, M. The liposoluble proteome of Mycoplasma agalactiae: an insight into the minimal protein complement of a bacterial membrane. BMC Microbiol. 2010; 10.

35. Corona L, Cillara G, and Tola S. Proteomic approach for identification of immunogenic proteins of Mycoplasma mycoides subsp capri. Vet Microbiol. 2013; 167: 434-439.

36. Nuyttens H, Cyncynatus C, Renaudin H, Pereyre S, and Bebear C. Identification, expression and serological evaluation of the recombinant ATP synthase beta subunit of Mycoplasma pneumoniae. BMC Microbiol. 2010; 10.

37. Ko KY, Kim JW, Her M, Kang S, Jung SC, Cho DH, and Kim JY. Immunogenic proteins of Brucella abortus to minimize cross reactions in brucellosis diagnosis. Vet Microbiol. 2012; 156: 374-380.

38. Zhao Z, Yan F, Ji W, Luo D, Liu X, Xing L, Duan Y, Yang P, Shi X, Li Z, and Wang X. Identification of immunoreactive proteins of Brucella melitensis by immunoproteomics. Sci China-Life Sci. 2011; 54: 880-887.

39. Dallo SF, Kannan TR, Blaylock MW, and Baseman JB. Elongation factor $\mathrm{Tu}$ and $\mathrm{E} 1$ beta subunit of pyruvate dehydrogenase complex act as fibronectin binding proteins in Mycoplasma pneumoniae. Mol Microbiol. 2002; 46: 1041-1051.

40. Caswell JL and Archambault M. Mycoplasma bovis pneumonia in cattle. Anim Health Res Rev. 2007; 8: 161186.

41. Petersen TN, Brunak S, Von Heijne G, and Nielsen H. SignalP 4.0: discriminating signal peptides from transmembrane regions. Nat Methods. 2011; 8: 785-786.

42. Bagos PG, Tsirigos KD, Liakopoulos TD, and Hamodrakas SJ. Prediction of lipoprotein signal peptides in Grampositive bacteria with a Hidden Markov Model. J Proteome Res. 2008; 7: 5082-5093.

43. Bendtsen JD, Jensen LJ, Blom N, Von Heijne G, and Brunak S. Feature-based prediction of non-classical and leaderless protein secretion. Protein Eng Des Sel. 2004; 17:
349-356.

44. Kannan TR, Krishnan M, Ramasamy K, Becker A, Pakhomova ON, Hart PJ, and Baseman JB. Functional mapping of community-acquired respiratory distress syndrome (CARDS) toxin of Mycoplasma pneumoniae defines regions with ADP-ribosyltransferase, vacuolating and receptor-binding activities. Mol Microbiol. 2014; 93: 568-581.

45. Lei S, Rui G, Zhengyan Y, Yong Z, Jie P, Zhibin HU, Lixia W, Changmin H, Tao L, Yingyu, C, Juanhong L, Junlong Z, Huanchun C, and Guo A. Diagnosis of Cattle Infectious Mycoplasma bovis Pneumonia. J HZAU. 2008; 27: 629633.

46. Bai ZD, Shi L, Hu CM., Chen X, Qi JJ, Ba XL, Peng QJ, Chen YY, Chen HC, and Guo A. Development of a loopmediated isothermal amplification assay for sensitive and rapid detection of Mycoplasma bovis. African J Biotech. 2011; 10: 12333-12338.

47. Zhang A, Xie C, Chen H, and Jin M. Identification of immunogenic cell wall-associated proteins of Streptococcus suis serotype 2. Proteomics. 2008; 8: 3506-3515.

48. Parraga-Nino N, Colome-Calls N, Canals F, Querol E, and Ferrer-Navarro M. A Comprehensive Proteome of Mycoplasma genitalium. J Proteome Res. 2012; 11: 33053316.

49. Han X, Khan FA, Zhu X, Zhang R, Mustafa R, Hu C, Chen Y, Chen H, and Guo A. Establishment of an antibody avidity test to differentiate vaccinated cattle from those naturally infected with Mycoplasma bovis. Vet J. 2015; 203: 79-84.

50. Weiss W and Gorg A. High-resolution two-dimensional electrophoresis. Methods Mol Biol. 2009; 564: 13-32.

51. Arnold K, Bordoli L, Kopp J, and Schwede T. The SWISSMODEL workspace: a web-based environment for protein structure homology modelling. Bioinformatics. 2006; 22 : 195-201.

52. Ponomarenko J, Bui HH, Li W, Fusseder N, Bourne PE, Sette A, and Peters B. ElliPro: a new structure-based tool for the prediction of antibody epitopes. BMC Bioinformatics. 2008; 9.

53. Vizcaíno JA, Csordas A, Del-Toro N, Dianes JA, Griss J, Lavidas I, Mayer G, Perez-Riverol Y, Reisinger F, Ternent T, Xu QW, Wang R, and Hermjakob H. 2016 update of the PRIDE database and related tools. Nucleic Acids Res. 2016; 44: 447-456. 